\title{
ON A PARAMETRIC NONLINEAR DIRICHLET PROBLEM WITH SUBDIFFUSIVE AND EQUIDIFFUSIVE REACTION
}

\author{
NIKOLAOS S. PAPAGEORGIOU AND PATRICK WINKERT
}

\begin{abstract}
We study a nonlinear parametric elliptic equation (nonlinear eigenvalue problem) driven by a nonhomogeneous differential operator. Our setting incorporates equations driven by the $p$-Laplacian, the $(p, q)$-Laplacian, and the generalized $p$-mean curvature differential operator. Applying variational methods we show that for $\lambda>0$ (the parameter) sufficiently large the problem has at least three nontrivial smooth solutions whereby one is positive, one is negative and the last one has changing sign (nodal). In the particular case of $(p, 2)$-equations, using Morse theory, we produce another nodal solution for a total of four nontrivial smooth solutions.
\end{abstract}

\section{INTRODUCTION}

Let $\Omega \subseteq \mathbb{R}^{N}$ be a bounded domain with a $C^{2}$-boundary $\partial \Omega$ and let $1<q \leq p$. We study the following nonlinear nonhomogeneous eigenvalue problem

$$
\begin{aligned}
-\operatorname{div} a(\nabla u) & =\lambda|u|^{q-2} u-f(x, u) & & \text { in } \Omega, \\
u & =0 & & \text { on } \partial \Omega,
\end{aligned}
$$

where the mapping $a: \mathbb{R}^{N} \rightarrow \mathbb{R}^{N}$ is supposed to be continuous and strictly monotone with $(p-1)$-growth. The precise regularity conditions are presented in hypotheses H(a) (see Section 2). These conditions incorporate in our framework of analysis some important differential operators, such as the $p$-Laplace differential operator $(1<p<\infty)$, the $(p, q)$-differential operator $(1<q<p)$, and the generalized $p$-mean curvature differential operator $(1<p<\infty)$. Furthermore, $\lambda>0$ is a parameter to be specified later and the perturbation $f: \Omega \times \mathbb{R} \rightarrow \mathbb{R}$ is a Carathéodory function (i.e., $x \mapsto f(x, s)$ is measurable for all $s \in \mathbb{R}$ and $s \mapsto f(x, s)$ is continuous for a.a. $x \in \Omega)$ whereby it is assumed that $f(x, \cdot)$ exhibits a $(p-1)$-superlinear growth near $\pm \infty$ for a.a. $x \in \Omega$. We note that in case $f(x, s)=f(s)=|s|^{r-2} s$, $q \leq p<r<\infty$, and $-\operatorname{div} a(\nabla(\cdot))=-\Delta_{p}$ (the negative $p$-Laplacian), problem $(P)_{\lambda}$ becomes the so-called $p$-logistic equation which is important in mathematical biology and describes the dynamics of biological populations whose mobility is density-dependent. If $q<p$, then we have the subdiffusive case while the equidiffusive case occurs for $q=p$. Since the solution of these applications describes population density, positive solutions are of concern (see Gurtin-MacCamy [24]). Our aim is to prove a multiplicity theorem for problem $(P)_{\lambda}$ describing the number of solutions as the parameter $\lambda>0$ varies. Moreover, we provide sign information for all the solutions produced.

2010 Mathematics Subject Classification. 35J20, 35J60, 35J92, 58E05.

Key words and phrases. Constant sign and nodal solutions, nonlinear regularity, critical point of mountain pass type, extremal solutions, local minimizers, maximum principle. 
Problem $(P)_{\lambda}$ was first studied by Ambrosetti-Lupo [2], Ambrosetti-Mancini [3], and Struwe [32], [33, p. 147]. In all these works the differential operator involved is the Laplacian (i.e. $a(\xi)=\xi$ for all $\xi \in \mathbb{R}^{N}$ ) and the perturbation $f$ is $x$-independent satisfying either $f \in C^{1}(\mathbb{R})$ (see [2], [3], [32]) or $f$ is Lipschitz continuous (see [33]). Therein, it is proved that if $\lambda>\tilde{\lambda}_{2}$, where $\tilde{\lambda}_{2}$ denotes the second eigenvalue of $\left(-\Delta, H_{0}^{1}(\Omega)\right)$, then the problem has at least three nontrivial solutions. However, they do not provide sign information for these solutions. An extension to the study of $p$-Laplacian equations $(1<p<\infty)$ was done by Averna-Motreanu-Marano [5], respectively, by Papageorgiou-Papageorgiou [29]. In the present paper we prove a similar three-solutions-theorem for problem $(P)_{\lambda}$ providing sign information for all solutions obtained. Moreover, in the particular case of $(p, 2)$-equations (i.e., $a(\xi)=\|\xi\|^{p-2} \xi+\xi$ for all $\xi \in \mathbb{R}^{N}$ with $2<p<\infty$ is the sum of a $p$-Laplacian and a Laplacian), we obtain the existence of four nontrivial solutions with complete sign information. In fact, as the results of Dancer [16] suggest (see also Struwe [33, p. 147]), one cannot expect more than four nontrivial solutions without any symmetry condition on the perturbation, even for $\lambda>0$ large.

Our approach is variational based on the critical point theory coupled with the usage of suitable perturbation and truncation techniques and with comparison principles. In the last section we also employ Morse theory (critical groups) dealing with the special case of the $(p, 2)$-equation. The main mathematical tools which will be used in this paper are recalled in the next section for the convenience of the reader.

\section{MATHEMATICAL BACKGROUND AND HYPOTHESES}

We start with some basic definitions and facts about critical point theory which we will need in the sequel. Let $X$ be a Banach space and $X^{*}$ its topological dual while $\langle\cdot, \cdot\rangle$ is taken for the duality brackets to the pair $\left(X^{*}, X\right)$. We have the following definition.

Definition 2.1. The functional $\varphi \in C^{1}(X)$ fulfills the Palais-Smale condition at the level $c \in \mathbb{R}$ (the $P S_{c}$-condition for short) if every sequence $\left(u_{n}\right)_{n \geq 1} \subseteq X$ satisfying $\varphi\left(u_{n}\right) \rightarrow c$ and $\varphi^{\prime}\left(u_{n}\right) \rightarrow 0$ in $X^{*}$, admits a strongly convergent subsequence. We say that $\varphi$ satisfies the Palais-Smale condition (the PS-condition for short) if it satisfies the $P S_{c}$-condition for every $c \in \mathbb{R}$.

Using this compactness-type condition, we can prove the following minimax theorem known in the literature as the Mountain Pass Theorem.

Theorem 2.2. If $\varphi \in C^{1}(X), u_{1}, u_{2} \in X,\left\|u_{2}-u_{1}\right\|>\rho>0$,

$$
\max \left\{\varphi\left(u_{1}\right), \varphi\left(u_{2}\right)\right\}<\inf \left\{\varphi(u):\left\|u-u_{1}\right\|=\rho\right\}=: \eta_{\rho}
$$

and $\varphi$ satisfies the $P S_{c}$-condition where

$$
c=\inf _{\gamma \in \Gamma} \max _{0 \leq t \leq 1} \varphi(\gamma(t))
$$

with $\Gamma=\left\{\gamma \in C([0,1], X): \gamma(0)=u_{1}, \gamma(1)=u_{2}\right\}$, then $c \geq \eta_{\rho}$ and $c$ is a critical value of $\varphi$.

Given $\varphi \in C^{1}(X)$ and $c \in \mathbb{R}$, we introduce the following sets

$$
\begin{array}{ll}
\varphi^{c}=\{u \in X: \varphi(u) \leq c\} & \text { (the sublevel set of } \varphi \text { at } c), \\
K_{\varphi}=\left\{u \in X: \varphi^{\prime}(u)=0\right\} & \text { (the critical set of } \varphi \text { ), } \\
K_{\varphi}^{c}=\left\{u \in K_{\varphi}: \varphi(u)=c\right\} & \text { (the critical set of } \varphi \text { at the level } c \text { ). }
\end{array}
$$


Another result from critical point theory, which will be needed, is the so-called Second Deformation Lemma (see, for example, Gasiński-Papageorgiou [23, p. 628]).

Lemma 2.3. If $\varphi \in C^{1}(X), a \in \mathbb{R}, a<b \leq+\infty, \varphi$ satisfies the $P S_{c}$-condition for every $c \in[a, b), \varphi$ has no critical values in $(a, b)$ and $\varphi^{-1}(a)$ contains at most a finite number of critical points of $\varphi$, then there exists a continuous map $h$ : $[0,1] \times\left(\varphi^{b} \backslash K_{\varphi}^{b}\right) \rightarrow \varphi^{b}$ such that

(a) $h(0, u)=u$ for all $u \in \varphi^{b} \backslash K_{\varphi}^{b}$;

(b) $h\left(1, \varphi^{b} \backslash K_{\varphi}^{b}\right) \subseteq \varphi^{a}$;

(c) $h(t, u)=u$ for all $(t, u) \in[0,1] \times \varphi^{a}$;

(d) $\varphi(h(t, u)) \leq \varphi(h(s, u))$ for all $t, s \in[0,1], s \leq t$, and all $u \in \varphi^{b} \backslash K_{\varphi}^{b}$.

Remark 2.4. In particular, Lemma 2.3 implies that $\varphi^{a}$ is a strong deformation retract at $\varphi^{b} \backslash K_{\varphi}^{b}$.

As usual, we denote by $C_{0}^{1}(\bar{\Omega})$ the ordered Banach space

$$
C_{0}^{1}(\bar{\Omega})=\left\{u \in C^{1}(\bar{\Omega}):\left.u\right|_{\partial \Omega}=0\right\}
$$

with positive cone

$$
C_{0}^{1}(\bar{\Omega})_{+}=\left\{u \in C_{0}^{1}(\bar{\Omega}): u(x) \geq 0 \forall x \in \bar{\Omega}\right\},
$$

which has a nonempty interior given by

$$
\operatorname{int}\left(C_{0}^{1}(\bar{\Omega})_{+}\right)=\left\{u \in C_{0}^{1}(\bar{\Omega}): u(x)>0 \forall x \in \Omega \text { and } \frac{\partial u}{\partial n}(x)<0 \forall x \in \partial \Omega\right\},
$$

where $n=n(x)$ is the outer unit normal at $x \in \partial \Omega$.

Let $g, h \in L^{\infty}(\Omega)$. We write $g \prec h$ if for every compact set $K \subseteq \Omega$ there exists $\varepsilon>0$ such that $g(x)+\varepsilon \leq h(x)$ for a.a. $x \in K$. Clearly, if $g, h \in C(\Omega)$ and $g(x)<h(x)$ for all $x \in \Omega$, then $g \prec h$.

In order to accommodate the extra linear term $-\Delta u$, an easy modification of the proof of Lemma 3.7 in Filippakis-O'Regan-Papageorgiou [20] (see also Arcoya-Ruiz [4, Proposition 2.6]) leads to the following strong comparison principle.

Proposition 2.5. Let $\varepsilon \geq 0, p \leq r<\infty$, and $g, h \in L^{\infty}(\Omega)$. If $u, v \in C_{0}^{1}(\bar{\Omega})$ are solutions of

$$
\begin{aligned}
& -\Delta_{p} u-\Delta u+\varepsilon|u|^{r-2} u=g \quad \text { in } \Omega, \\
& -\Delta_{p} v-\Delta v+\varepsilon|v|^{r-2} v=h \quad \text { in } \Omega,
\end{aligned}
$$

and $v \in \operatorname{int}\left(C_{0}^{1}(\bar{\Omega})_{+}\right)$, then $v-u \in \operatorname{int}\left(C_{0}^{1}(\bar{\Omega})_{+}\right)$.

Now, we are ready to introduce the hypotheses on the map $a(\cdot)$. Let $\vartheta \in$ $C^{1}(0,+\infty)$ be a function satisfying

$$
0<\hat{c} \leq \frac{t \vartheta^{\prime}(t)}{\vartheta(t)} \leq c_{0} \quad \text { and } \quad c_{1} t^{p-1} \leq \vartheta(t) \leq c_{2}\left(1+t^{p-1}\right)
$$

for all $t>0$ and with some constants $\hat{c}, c_{0}, c_{1}, c_{2}>0$.

Then the hypotheses on $a(\cdot)$ are the following.

$\mathrm{H}(\mathrm{a}): a(\xi)=a_{0}(\|\xi\|) \xi$ for all $\xi \in \mathbb{R}^{N}$ with $a_{0}(t)>0$ for all $t>0$ and 
(i) $a_{0} \in C^{1}(0, \infty), t \mapsto t a_{0}(t)$ is strictly increasing, $\lim _{t \rightarrow 0^{+}} t a_{0}(t)=0$, and

$$
\lim _{t \rightarrow 0^{+}} \frac{t a_{0}^{\prime}(t)}{a_{0}(t)}>-1
$$

(ii) $\|\nabla a(\xi)\| \leq c_{3} \frac{\vartheta(\|\xi\|)}{\|\xi\|}$ for all $\xi \in \mathbb{R}^{N} \backslash\{0\}$ and some $c_{3}>0$;

(iii) $(\nabla a(\xi) y, y)_{\mathbb{R}^{N}} \geq \frac{\vartheta(\|\xi\|)}{\|\xi\|}\|y\|^{2}$ for all $\xi \in \mathbb{R}^{N} \backslash\{0\}$ and all $y \in \mathbb{R}^{N}$.

Remark 2.6. From hypothesis $\mathrm{H}(\mathrm{a})(\mathrm{i})$ we see at once that $a \in C^{1}\left(\mathbb{R}^{N} \backslash\{0\}, \mathbb{R}^{N}\right) \cap$ $C\left(\mathbb{R}^{N}, \mathbb{R}^{N}\right)$ implying that hypotheses $\mathrm{H}(\mathrm{a})(\mathrm{ii})$, (iii) make sense. Let $G_{0}(t)=$ $\int_{0}^{t} s a_{0}(s) d s$ and consider the function $G: \mathbb{R}^{N} \rightarrow \mathbb{R}$ defined by $G(\xi)=G_{0}(\|\xi\|)$ for all $\xi \in \mathbb{R}^{N}$. Then

$$
\nabla G(\xi)=G_{0}^{\prime}(\|\xi\|) \frac{\xi}{\|\xi\|}=a_{0}(\|\xi\|) \xi=a(\xi) \quad \text { for all } \xi \in \mathbb{R}^{N} \backslash\{0\} .
$$

Note that by virtue of hypothesis $\mathrm{H}(\mathrm{a})(\mathrm{i})$, we have $\nabla G(0)=0$ as well. Evidently $G(\cdot)$ is convex and $G(0)=0, \nabla G(\xi)=a(\xi)$ for all $\xi \in \mathbb{R}^{N}$. Hence we have the estimate

$$
G(\xi) \leq(a(\xi), \xi)_{\mathbb{R}^{N}} \quad \text { for all } \xi \in \mathbb{R}^{N} .
$$

The hypotheses H(a), (2.1) and the integral form of the mean value theorem lead to the following lemma, which summarizes the main properties of the map $a(\cdot)$.

Lemma 2.7. Let the hypotheses $H(a)$ be satisfied. Then, there hold

(a) $\xi \rightarrow a(\xi)$ is maximal monotone and strictly monotone;

(b) $\|a(\xi)\| \leq c_{4}\left(1+\|\xi\|^{p-1}\right)$ for all $\xi \in \mathbb{R}^{N}$ and some $c_{4}>0$;

(c) $(a(\xi), \xi)_{\mathbb{R}^{N}} \geq \frac{c_{1}}{p-1}\|\xi\|^{p}$ for all $\xi \in \mathbb{R}^{N}$.

As a direct consequence of Lemma 2.7 we obtain the following growth estimates for the potential $G(\cdot)$.

Corollary 2.8. If hypotheses $H(a)$ hold, then

$$
\frac{c_{1}}{p(p-1)}\|\xi\|^{p} \leq G(\xi) \leq c_{5}\left(1+\|\xi\|^{p}\right) \quad \text { for all } \xi \in \mathbb{R}^{N} \text { and some } c_{5}>0 \text {. }
$$

Example 2.9. The following maps satisfy hypotheses $H(a)$ :

(a) Let $a(\xi)=\|\xi\|^{p-2} \xi$ with $1<p<\infty$. This map corresponds to the $p$-Laplace differential operator defined by

$$
\Delta_{p} u=\operatorname{div}\left(\|\nabla u\|^{p-2} \nabla u\right) \quad \text { for all } u \in W_{0}^{1, p}(\Omega) .
$$

The potential is $G(\xi)=\frac{1}{p}\|\xi\|^{p}$ for all $\xi \in \mathbb{R}^{N}$.

(b) The function $a(\xi)=\|\xi\|^{p-2} \xi+\mu\|\xi\|^{q-2} \xi$ with $1<q<p$ and $\mu>0$ compares with the $(p, q)$-differential operator defined by $\Delta_{p} u+\mu \Delta_{q} u$ for all $u \in W_{0}^{1, p}(\Omega)$. The potential is $G(\xi)=\frac{1}{p}\|\xi\|^{p}+\frac{\mu}{q}\|\xi\|^{q}$ for all $\xi \in \mathbb{R}^{N}$. Equations driven by a $(p, q)$-differential operator arise in mathematical physics such as quantum physics (for existence of soliton solutions, see Benci-D'Avenia-FortunatoPisani [6]) and in plasma physics and biophysics (see Cherfils-Il'yasov [11]). Recently, there have been some existence and multiplicity results for such operators. We refer to the works of Cingolani-Degiovanni [12], Medeiros-Perera [27], Papageorgiou-Smyrlis [30], and Sun [34]. 
(c) If $a(\xi)=\left(1+\|\xi\|^{2}\right)^{\frac{p-2}{2}} \xi$ with $1<p<\infty$, then this map represents the generalized $p$-mean curvature differential operator defined by

$$
\operatorname{div}\left[\left(1+\|\nabla u\|^{2}\right)^{\frac{p-2}{2}} \nabla u\right] \quad \text { for all } u \in W_{0}^{1, p}(\Omega) .
$$

The potential is $G(\xi)=\frac{1}{p}\left[\left(1+\|\xi\|^{2}\right)^{\frac{p}{2}}-1\right]$ for all $\xi \in \mathbb{R}^{N}$.

(d) Let $a(\xi)=\|\xi\|^{p-2} \xi \pm \frac{\|\xi\|^{p-2} \xi}{1+\|\xi\|^{p}}$ with $2 \leq p<\infty$. Then, the potential is given by $G(\xi)=\frac{1}{p}\|\xi\|^{p} \pm \frac{1}{p} \ln \left(1+\|\xi\|^{p}\right)$ for all $\xi \in \mathbb{R}^{N}$. It should be mentioned the work of Clément-García-Huidobro-Manásevich-Schmitt [14] where the authors also consider nonlinear nonhomogeneous equations using tools from the theory of Orlicz spaces producing solutions of constant sign for a different nonlinearity than ours.

Now, let $f_{0}: \Omega \times \mathbb{R} \rightarrow \mathbb{R}$ be a Carathéodory function with subcritical growth in the second argument, that is

$$
\left|f_{0}(x, s)\right| \leq a(x)+c|s|^{r-1} \quad \text { for a.a. } x \in \Omega \text {, for all } s \in \mathbb{R},
$$

with $a \in L^{\infty}(\Omega)_{+}, c>0$, and $1<r<p^{*}$, where $p^{*}$ is the critical exponent of $p$ given by

$$
p^{*}= \begin{cases}\frac{N p}{N-p} & \text { if } p<N, \\ +\infty & \text { if } p \geq N .\end{cases}
$$

Let $F_{0}(x, s)=\int_{0}^{s} f_{0}(x, t) d t$ and let $\varphi_{0}: W_{0}^{1, p}(\Omega) \rightarrow \mathbb{R}$ be the $C^{1}$-functional defined by

$$
\varphi_{0}(u)=\int_{\Omega} G(\nabla u) d x-\int_{\Omega} F_{0}(x, u) d x \quad \text { for all } u \in W_{0}^{1, p}(\Omega) .
$$

The following result is essentially due to Motreanu-Papageorgiou [28] (see also Brezis-Nirenberg [7], García Azorero-Peral Alonso-Manfredi [21] and Winkert [35] for earlier results in this direction). The result is a consequence of the nonlinear regularity theory (see [25], [26]).

Proposition 2.10. Let the assumptions in $H(a)$ be satisfied. If $u_{0} \in W_{0}^{1, p}(\Omega)$ is a local $C_{0}^{1}(\bar{\Omega})$-minimizer of $\varphi_{0}$, i.e., there exists $\rho_{0}>0$ such that

$$
\varphi_{0}\left(u_{0}\right) \leq \varphi_{0}\left(u_{0}+h\right) \quad \text { for all } h \in C_{0}^{1}(\bar{\Omega}) \text { with }\|h\|_{C_{0}^{1}(\bar{\Omega})} \leq \rho_{0},
$$

then $u_{0} \in C_{0}^{1, \beta}(\bar{\Omega})$ for some $\beta \in(0,1)$ and $u_{0}$ is also a local $W_{0}^{1, p}(\Omega)$-minimizer of $\varphi_{0}$, i.e., there exists $\rho_{1}>0$ such that

$$
\varphi_{0}\left(u_{0}\right) \leq \varphi_{0}\left(u_{0}+h\right) \quad \text { for all } h \in W_{0}^{1, p}(\Omega) \text { with }\|h\|_{W_{0}^{1, p}(\Omega)} \leq \rho_{1} .
$$
by

Let $\frac{1}{p}+\frac{1}{p^{\prime}}=1$ and let $A: W_{0}^{1, p}(\Omega) \rightarrow W^{-1, p^{\prime}}(\Omega)$ be the nonlinear map defined

$$
\langle A(u), v\rangle=\int_{\Omega}(a(\nabla u), \nabla v)_{\mathbb{R}^{N}} d x \quad \text { for all } u, v \in W_{0}^{1, p}(\Omega) .
$$

The following result summarizes the basic properties of $A$ (see, for example, Gasiński-

Papageorgiou [22, p. 562]). 
Proposition 2.11. If hypotheses $H(a)$ hold, then $A: W_{0}^{1, p}(\Omega) \rightarrow W^{-1, p^{\prime}}(\Omega)$ defined by (2.3) is continuous, monotone (hence maximal monotone), and of type $(S)_{+}$, i.e., if $u_{n} \rightarrow u$ in $W_{0}^{1, p}(\Omega)$ and $\lim \sup _{n \rightarrow \infty}\left\langle A\left(u_{n}\right), u_{n}-u\right\rangle \leq 0$, then $u_{n} \rightarrow u$ in $W_{0}^{1, p}(\Omega)$.

By $\Delta_{r}, 1<r<\infty$, we denote the special case of the map above corresponding to the $r$-Laplacian, i.e.,

$$
\left\langle\Delta_{r}(u), v\right\rangle=\int_{\Omega}\|\nabla u\|^{r-2}(\nabla u, \nabla v)_{\mathbb{R}^{N}} d x \quad \text { for all } u, v \in W_{0}^{1, r}(\Omega) .
$$

If $r=2$, it reduces to the Laplacian and we write $\Delta_{2}=\Delta \in \mathcal{L}\left(H_{0}^{1}(\Omega), H^{-1}(\Omega)\right)$.

Next let us recall some basic facts about the spectrum of the negative Dirichlet $r$-Laplacian, i.e., of $\left(-\Delta_{r}, W_{0}^{1, r}(\Omega)\right)$ with $1<r<\infty$. We consider the following nonlinear eigenvalue problem

$$
\begin{aligned}
-\Delta_{r} u & =\hat{\lambda}|u|^{r-2} u & & \text { in } \Omega, \\
u & =0 & & \text { on } \partial \Omega .
\end{aligned}
$$

A number $\hat{\lambda} \in \mathbb{R}$ is an eigenvalue of $\left(-\Delta_{r}, W_{0}^{1, r}(\Omega)\right)$ if problem (2.4) admits a nontrivial solution $\hat{u} \in W_{0}^{1, p}(\Omega)$ which is called an eigenfunction corresponding to the eigenvalue $\hat{\lambda}$. By $\hat{\sigma}(r)$ we denote the set of eigenvalues of $\left(-\Delta_{r}, W_{0}^{1, r}(\Omega)\right)$. It is known that the set $\hat{\sigma}(r)$ has a smallest element $\hat{\lambda}_{1}(r)$, which has the following properties:

- $\hat{\lambda}_{1}(r)$ is positive;

- $\hat{\lambda}_{1}(r)$ is isolated, that is, there exists $\varepsilon>0$ such that $\left[\hat{\lambda}_{1}(r), \hat{\lambda}_{1}(r)+\varepsilon\right) \cap$ $\hat{\sigma}(r)=\hat{\lambda}_{1}(r)$;

- $\hat{\lambda}_{1}(r)$ is simple, that is, if $u, v$ are two eigenfunctions corresponding to $\hat{\lambda}_{1}(r)$, then $u=k v$ for some $k \in \mathbb{R}$;

$$
\hat{\lambda}_{1}(r)=\inf \left[\frac{\|\nabla u\|_{L^{r}(\Omega)}^{r}}{\|u\|_{L^{r}(\Omega)}^{r}}: u \in W_{0}^{1, r}(\Omega), u \neq 0\right] .
$$

In (2.5) the infimum is realized on the corresponding one dimensional eigenspace. Regarding (2.5) it is also clear that the eigenfunctions corresponding to $\hat{\lambda}_{1}(r)$ do not change sign. In fact it turns out that $\hat{\lambda}(r)$ is the only eigenvalue with eigenfunctions of constant sign. All the other eigenvalues have eigenfunctions which are nodal (i.e., sign changing). In what follows by $\hat{u}_{1}(r)$ we denote the $L^{r}$ normalized (i.e., $\left\|\hat{u}_{1}(r)\right\|_{L^{r}(\Omega)}=1$ ) positive eigenfunction corresponding to $\hat{\lambda}_{1}(r)$. The nonlinear regularity theory and the nonlinear maximum principle imply that $\hat{u}_{1}(r) \in \operatorname{int}\left(C_{0}^{1}(\bar{\Omega})_{+}\right)$(see [25], [26] and [23, pp. 737-738]). Furthermore, the set $\hat{\sigma}(r)$ is closed and since $\hat{\lambda}_{1}(r)$ is isolated the second eigenvalue $\hat{\lambda}_{2}(r)$ is well-defined by

$$
\hat{\lambda}_{2}(r)=\inf \left[\hat{\lambda} \in \hat{\sigma}(r): \hat{\lambda}>\hat{\lambda}_{1}(r)\right] .
$$


Now, let $\partial B_{1}^{L^{r}}=\left\{u \in L^{r}(\Omega):\|u\|_{L^{r}(\Omega)}=1\right\}$ and $M_{r}=W_{0}^{1, r}(\Omega) \cap \partial B_{1}^{L^{r}}$. Then, $\hat{\lambda}_{2}(r)$ admits the following variational characterization (see Cuesta-de FigueiredoGossez [15]).

Proposition 2.12. There holds

$$
\hat{\lambda}_{2}(r)=\inf _{\hat{\gamma} \in \hat{\Gamma}(r)} \max _{-1 \leq t \leq 1}\|\nabla \hat{\gamma}(t)\|_{L^{r}(\Omega)}^{r},
$$

where $\hat{\Gamma}(r)=\left\{\hat{\gamma} \in C\left([-1,1], M_{r}\right): \hat{\gamma}(-1)=-\hat{u}_{1}(r), \hat{\gamma}(1)=\hat{u}_{1}(r)\right\}$.

The Lusternik-Schnirelmann minimax scheme gives a whole strictly increasing sequence $\left(\hat{\lambda}_{k}(r)\right)_{k \geq 1}$ of eigenvalues, but it is not known if this sequence exhausts the whole set $\hat{\sigma}(r)$. Indeed, this is true if $N=1$ (ordinary differential equations) or if $r=2$ (linear eigenvalue problem). In the latter case by $E\left(\hat{\lambda}_{k}(2)\right), k \geq 1$, we denote the eigenspace corresponding to the eigenvalue $\hat{\lambda}_{k}(2), k \geq 1$.

Next, let us recall some basic facts about Morse theory. Let $X$ be a Banach space and let $\left(Y_{1}, Y_{2}\right)$ be a topological pair such that $Y_{2} \subseteq Y_{1} \subseteq X$. For every integer $k \geq 0$, the term $H_{k}\left(Y_{1}, Y_{2}\right)$ stands for the $k \stackrel{t h}{=}$-relative singular homology group with integer coefficients. For $k<0$, we have $H_{k}\left(Y_{1}, Y_{2}\right)=0$. Let $\varphi \in C^{1}(X)$ and let $u \in X$ be an isolated critical point of $\varphi$ with $\varphi(u)=c$ (i.e., $u \in K_{\varphi}^{c}$ ). The critical groups of $\varphi$ at $u \in K_{\varphi}^{c}$ are defined by

$$
C_{k}(\varphi, u)=H_{k}\left(\varphi^{c} \cap U, \varphi^{c} \cap U \backslash\{u\}\right) \text { for all } k \geq 0,
$$

where $U$ is a neighborhood of $u$ such that $K_{\varphi} \cap \varphi^{c} \cap U=\{u\}$. The excision property of singular homology implies that this definition of critical groups is independent of the particular choice of the neighborhood $U$.

Suppose that $\varphi \in C^{1}(X)$ satisfies the PS-condition and $\inf \varphi\left(K_{\varphi}\right)>-\infty$. Let $c<\inf \varphi\left(K_{\varphi}\right)$. The critical groups of $\varphi$ at infinity are defined by

$$
C_{k}(\varphi, \infty)=H_{k}\left(X, \varphi^{c}\right) \text { for all } k \geq 0 .
$$

This definition is independent of the choice of the level $c<\inf \varphi\left(K_{\varphi}\right)$ which is a consequence of the Second Deformation Lemma stated in Lemma 2.3.

We assume that $K_{\varphi}$ is finite and introduce the following series in $t \in \mathbb{R}$ :

$$
\begin{aligned}
& M(t, u)=\sum_{k \geq 0} \operatorname{rank} C_{k}(\varphi, u) t^{k} \quad \text { for all } u \in K_{\varphi}, \\
& P(t, \infty)=\sum_{k \geq 0} \operatorname{rank} C_{k}(\varphi, \infty) t^{k} .
\end{aligned}
$$

Then, the Morse relation reads as follows:

$$
\sum_{u \in K_{\varphi}} M(t, u)=P(t, \infty)+(1+t) Q(t) \quad \text { for all } t \in \mathbb{R},
$$

where $Q(t)$ is a formal series in $t \in \mathbb{R}$ with nonnegative integer coefficients.

It is well-known that if a functional satisfies the PS-condition or the C-condition and it is bounded below, then it is coercive (see Caklović-Li-Willem [9] and GasińskiPapageorgiou [23, p. 614]). The converse is in general not true. However, in the setting of this work the converse is true. 
More precisely, let $\hat{f}: \Omega \times \mathbb{R} \rightarrow \mathbb{R}$ be a Carathéodory function such that

$$
|\hat{f}(x, s)| \leq a(x)\left(1+|s|^{r-1}\right) \quad \text { for a.a. } x \in \Omega, \text { for all } s \in \mathbb{R},
$$

with $a \in L^{\infty}(\Omega)_{+}$, and $p \leq r<p^{*}$. We set $\hat{F}(x, s)=\int_{0}^{s} \hat{f}(x, t) d t$ and consider the $C^{1}$-functional $\hat{\varphi}: W_{0}^{1, p}(\Omega) \rightarrow \mathbb{R}$ defined by

$$
\hat{\varphi}(u)=\int_{\Omega} G(\nabla u) d x-\int_{\Omega} \hat{F}(x, u) d x .
$$

Proposition 2.13. If $\hat{\varphi}$ is coercive, then it satisfies the PS-condition.

Proof. Suppose $\left(u_{n}\right)_{n \geq 1} \subseteq W_{0}^{1, p}(\Omega)$ is a PS-sequence, that is

$$
\begin{aligned}
& \left|\hat{\varphi}\left(u_{n}\right)\right| \leq \tilde{M} \text { for some } \tilde{M}>0, \text { for all } n \geq 1, \\
& \hat{\varphi}^{\prime}\left(u_{n}\right) \rightarrow 0 \text { in } W^{-1, p^{\prime}}(\Omega) .
\end{aligned}
$$

The statement in (2.7) along with the coercivity of $\hat{\varphi}$ implies that $\left(u_{n}\right)_{n \geq 1} \subseteq$ $W_{0}^{1, p}(\Omega)$ is bounded. Therefore, we may assume that

$$
u_{n} \rightarrow u \quad \text { in } W_{0}^{1, p}(\Omega) \quad \text { and } \quad u_{n} \rightarrow u \quad \text { in } L^{p}(\Omega) .
$$

From (2.8) it follows

$$
\left|\left\langle A\left(u_{n}\right), v\right\rangle-\int_{\Omega} \hat{f}\left(x, u_{n}\right) v d x\right| \leq \frac{\varepsilon_{n}\|v\|_{W_{0}^{1, p}(\Omega)}}{1+\left\|u_{n}\right\|_{W_{0}^{1, p}(\Omega)}},
$$

for all $v \in W_{0}^{1, p}(\Omega)$ with $\varepsilon_{n} \rightarrow 0^{+}$. Now, choosing $v=u_{n}-u$, passing to the limit as $n \rightarrow \infty$, and using the convergence properties in (2.9) we obtain

$$
\lim _{n \rightarrow \infty}\left\langle A\left(u_{n}\right), u_{n}-u\right\rangle=0,
$$

which by the $(\mathrm{S})_{+}$-property of $A$ (see Proposition 2.11) gives $u_{n} \rightarrow u$ in $W_{0}^{1, p}(\Omega)$. Hence, $\hat{\varphi}$ fulfills the PS-condition.

Finally we conclude this section by fixing our notation. Throughout this paper we denote the norm of $W_{0}^{1, p}(\Omega)$ through $\|\cdot\|_{W_{0}^{1, p}(\Omega)}$ and thanks to Poincare's inequality we have $\|u\|_{W_{0}^{1, p}(\Omega)}=\|\nabla u\|_{L^{p}(\Omega)}$ for all $u \in W_{0}^{1, p}(\Omega)$. The norm of $\mathbb{R}^{N}$ is denoted by $\|\cdot\|$ and $(\cdot, \cdot)_{\mathbb{R}^{N}}$ stands for the inner product in $\mathbb{R}^{N}$. For $s \in \mathbb{R}$, we set $s^{ \pm}=\max \{ \pm s, 0\}$ and for $u \in W_{0}^{1, p}(\Omega)$ we define $u^{ \pm}(\cdot)=u(\cdot)^{ \pm}$. It is well known that

$$
u^{ \pm} \in W_{0}^{1, p}(\Omega), \quad|u|=u^{+}+u^{-}, \quad u=u^{+}-u^{-} .
$$

The Lebesgue measure on $\mathbb{R}^{N}$ is given by $|\cdot|_{N}$. Finally, for any Carathéodory function $h: \Omega \times \mathbb{R} \rightarrow \mathbb{R}$, we define the Nemytskij operator corresponding to the function $h$ by

$$
N_{h}(u)(\cdot)=h(\cdot, u(\cdot)) \quad \text { for all } u \in W_{0}^{1, p}(\Omega) .
$$




\section{Three SOlutions}

This section is devoted to the study of problem $(P)_{\lambda}$ with $\lambda>0$ appropriately large. We prove the existence of at least three nontrivial smooth solutions including complete sign information for these solutions. Precisely, it will be shown that the first solution is positive, the second one is negative, and the last one has changing sign (nodal).

Before we start with our results we state some stronger hypotheses on the map $a(\cdot)$ as in $\mathrm{H}(\mathrm{a})$ which will be needed in our proofs.

$\mathrm{H}(\mathrm{a})^{\prime}: a(\xi)=a_{0}(\|\xi\|) \xi$ for all $\xi \in \mathbb{R}^{N}$ with $a_{0}(t)>0$ for all $t>0$, hypotheses $\mathrm{H}(\mathrm{a})^{\prime}(\mathrm{i})-(\mathrm{iii})$ are the same as the corresponding hypotheses $\mathrm{H}(\mathrm{a})(\mathrm{i})-(\mathrm{iii})$ and

(iv) if $G_{0}(t)=\int_{0}^{t} s a_{0}(s) d s$ for all $t>0$, then $t \mapsto G_{0}\left(t^{\frac{1}{q}}\right)$ is convex in $(0,+\infty)$ and

$$
\limsup _{t \rightarrow 0^{+}} \frac{G_{0}(t)}{t^{q}}<+\infty
$$

Remark 3.1. The examples presented in Section 2 still satisfy hypotheses H(a)'. Note that hypothesis $\mathrm{H}(\mathrm{a})$ '(iv) implies

$$
G(\xi) \leq c_{6}\left(\|\xi\|^{q}+\|\xi\|^{p}\right) \quad \text { for all } \xi \in \mathbb{R}^{N},
$$

with some $c_{6}>0$.

The hypotheses on the perturbation $f$ are the following:

$\mathrm{H}(\mathrm{f})_{1}: f: \Omega \times \mathbb{R} \rightarrow \mathbb{R}$ is a Carathéodory function such that

(i) for every $\rho>0$, there exists $a_{\rho} \in L^{\infty}(\Omega)_{+}$such that

$$
|f(x, s)| \leq a_{\rho}(x) \text { for a.a. } x \in \Omega \text { and for all }|s| \leq \rho ;
$$

(ii) $\lim _{s \rightarrow \pm \infty} \frac{f(x, s)}{|s|^{p-2} s}=+\infty \quad$ uniformly for a.a. $x \in \Omega$;

(iii) $\lim _{s \rightarrow 0} \frac{f(x, s)}{|s|^{q-2} s}=0 \quad$ uniformly for a.a. $x \in \Omega$.

Remark 3.2. We point out that no growth restriction is imposed on $f(x, \cdot)$. This is in contrast to the considerations in [5] and [29], where it is required that $f(x, \cdot)$ has subcritical growth for a.a. $x \in \Omega$.

Example 3.3. The following functions satisfy hypotheses $H(f)_{1}$ :

$$
f_{1}(x, s)=a(x)|s|^{r-2} s \quad \text { and } \quad f_{2}(x, s)=a(x)|s|^{p-2} s \ln (1+|s|)
$$

with $a \in L^{\infty}(\Omega)$ and $p<r<\infty$.

Given any $\eta>0$, by virtue of hypothesis $\mathrm{H}(\mathrm{f})_{1}(\mathrm{ii})$, there exists $M_{1}=M_{1}(\eta)>1$ such that

$$
f(x, s) s \geq \eta|s|^{p} \quad \text { for a.a. } x \in \Omega \text { and for all }|s| \geq M_{1} .
$$

Let $\bar{u} \equiv \varsigma \in\left[M_{1},+\infty\right)$. Taking into account (3.2), $q \leq p$, and $M_{1}>1$, we conclude for $\eta=\lambda$

$$
0 \geq \lambda \bar{u}^{q-1}-f(x, \bar{u}) \quad \text { for a.a. } x \in \Omega \text {. }
$$


Similarly, if $\underline{v} \equiv-\varsigma$, then

$$
0 \leq \lambda|\underline{v}|^{q-2} \underline{v}-f(x, \underline{v}) \quad \text { for a.a. } x \in \Omega .
$$

Applying $\bar{u}$ and $\underline{v}$, we introduce the following truncations of the reaction in problem $(P)_{\lambda}$ :

$$
h_{\lambda}^{+}(x, s)= \begin{cases}0 & \text { if } s<0 \\ \lambda s^{q-1}-f(x, s) & \text { if } 0 \leq s \leq \bar{u} \\ \lambda \bar{u}^{q-1}-f(x, \bar{u}) & \text { if } \bar{u}<s\end{cases}
$$

and

$$
h_{\lambda}^{-}(x, s)= \begin{cases}\lambda|\underline{v}|^{q-2} \underline{v}-f(x, \underline{v}) & \text { if } s<\underline{v}, \\ \lambda|s|^{q-2} s-f(x, s) & \text { if } \underline{v} \leq s \leq 0, \\ 0 & \text { if } 0<s .\end{cases}
$$

It is easy to verify that both mappings are Carathéodory functions. We set $H_{\lambda}^{ \pm}(x, s)=$ $\int_{0}^{s} h_{\lambda}^{ \pm}(x, t) d t$ and consider the $C^{1}$-functionals $\hat{\varphi}_{\lambda}^{ \pm}: W_{0}^{1, p}(\Omega) \rightarrow \mathbb{R}$ defined by

$$
\hat{\varphi}_{\lambda}^{ \pm}(u)=\int_{\Omega} G(\nabla u) d x-\int_{\Omega} H_{\lambda}^{ \pm}(x, u) d x .
$$

Now, we are ready to produce two nontrivial constant sign solutions of $(P)_{\lambda}$ for suitable $\lambda>0$ large enough. In what follows, we use the notation

$$
\begin{aligned}
& {[0, \bar{u}]=\left\{u \in W_{0}^{1, p}(\Omega): 0 \leq u(x) \leq \bar{u} \text { a.e. in } \Omega\right\},} \\
& {[\underline{v}, 0]=\left\{u \in W_{0}^{1, p}(\Omega): \underline{v} \leq u(x) \leq 0 \text { a.e. in } \Omega\right\} .}
\end{aligned}
$$

Proposition 3.4. Suppose that hypotheses $H(a)$ ' and $H(f)_{1}$ are fulfilled and assume that

$$
\lambda> \begin{cases}q c_{6} \hat{\lambda}_{1}(q) & \text { if } q<p, \\ 2 q c_{6} \hat{\lambda}_{1}(q) & \text { if } q=p\end{cases}
$$

with the positive constant $c_{6}$ of Remark 3.1. Then problem $(P)_{\lambda}$ has at least two nontrivial constant sign solutions

$$
u_{0} \in[0, \bar{u}] \cap \operatorname{int}\left(C_{0}^{1}(\bar{\Omega})_{+}\right) \quad \text { and } \quad v_{0} \in[\underline{v}, 0] \cap\left(-\operatorname{int}\left(C_{0}^{1}(\bar{\Omega})_{+}\right)\right) .
$$

Proof. We start with the existence of the positive solution. Thanks to the truncation in (3.4) and Corollary 2.8, it is obvious that $\hat{\varphi}_{\lambda}^{+}$is coercive and taking into account the Sobolev embedding theorem we verify that $\hat{\varphi}_{\lambda}^{+}$is sequentially weakly lower semicontinuous as well. Hence, by virtue of the Weierstrass theorem, we find $u_{0} \in W_{0}^{1, p}(\Omega)$ such that

$$
\hat{\varphi}_{\lambda}^{+}\left(u_{0}\right)=\inf \left[\hat{\varphi}_{\lambda}^{+}(u): u \in W_{0}^{1, p}(\Omega)\right]=\hat{m}_{\lambda}^{+} .
$$

Given $\varepsilon>0$, from hypothesis $\mathrm{H}(\mathrm{f})(\mathrm{iii})$, we can find $\delta=\delta(\varepsilon) \in(0, \bar{u})$ such that

$$
F(x, s) \leq \frac{\varepsilon}{q}|s|^{q} \quad \text { for a.a. } x \in \Omega \text { and for all }|s| \leq \delta .
$$


Recalling $\hat{u}_{1}(q) \in \operatorname{int}\left(C_{0}^{1}(\bar{\Omega})_{+}\right)$, we choose $t \in(0,1)$ such that $t \hat{u}_{1}(q)(x) \in[0, \delta]$ for all $x \in \bar{\Omega}$. Then, due to (3.1), (3.4), (3.6) combined with $\left\|\hat{u}_{1}(q)\right\|_{L^{q}(\Omega)}=1$ and the fact that $\delta<\bar{u}$, we obtain

$$
\begin{aligned}
\hat{\varphi}_{\lambda}^{+}\left(t \hat{u}_{1}(q)\right) & =\int_{\Omega} G\left(\nabla\left(t \hat{u}_{1}(q)\right)\right) d x-\int_{\Omega} H_{\lambda}^{+}\left(x, t \hat{u}_{1}(q)\right) d x \\
& =\int_{\Omega} G\left(\nabla\left(t \hat{u}_{1}(q)\right)\right) d x-\frac{\lambda t^{q}}{q}\left\|\hat{u}_{1}(q)\right\|_{L^{q}(\Omega)}^{q}+\int_{\Omega} F\left(x, t \hat{u}_{1}(q)\right) d x \\
& \leq c_{6}\left(t^{q}\left\|\nabla\left(\hat{u}_{1}(q)\right)\right\|_{L^{q}(\Omega)}^{q}+t^{p}\left\|\nabla\left(\hat{u}_{1}(q)\right)\right\|_{L^{p}(\Omega)}^{p}\right)-\frac{\lambda t^{q}}{q}+\frac{\varepsilon t^{q}}{q} \\
& =c_{6}\left(t^{q} \hat{\lambda}_{1}(q)+t^{p}\left\|\nabla\left(\hat{u}_{1}(q)\right)\right\|_{L^{p}(\Omega)}^{p}\right)-\frac{\lambda-\varepsilon}{q} t^{q} \\
& =t^{q}\left[\frac{q c_{6} \hat{\lambda}_{1}(q)+\varepsilon-\lambda}{q}\right]+c_{6} t^{p}\left\|\nabla\left(\hat{u}_{1}(q)\right)\right\|_{L^{p}(\Omega)}^{p} .
\end{aligned}
$$

Now, we choose $\varepsilon$ such that

$$
0<\varepsilon< \begin{cases}\lambda-q c_{6} \hat{\lambda}_{1}(q) & \text { if } q<p \\ \lambda-2 q c_{6} \hat{\lambda}_{1}(q) & \text { if } q=p\end{cases}
$$

In both cases the right-hand side of (3.7) can be estimated above by zero. This gives (see also (3.5))

$$
\hat{\varphi}_{\lambda}^{+}\left(u_{0}\right)=\hat{m}_{\lambda}^{+}<0=\hat{\varphi}_{\lambda}^{+}(0)
$$

which means that $u_{0} \neq 0$ is nontrivial. Since $u_{0}$ is the global minimum of $\hat{\varphi}_{\lambda}^{+}$, we have $\left(\hat{\varphi}_{\lambda}^{+}\right)^{\prime}\left(u_{0}\right)=0$ which results in

$$
A\left(u_{0}\right)=N_{h_{\lambda}^{+}}\left(u_{0}\right)
$$

Taking $-u_{0}^{-} \in W_{0}^{1, p}(\Omega)$ as test function in (3.8) and applying Lemma 2.7(c) and (3.4), it follows

$$
\frac{c_{1}}{p-1}\left\|\nabla u_{0}^{-}\right\|_{L^{p}(\Omega)}^{p} \leq 0
$$

ensuring that $u_{0} \geq 0$. Next, we choose $\left(u_{0}-\bar{u}\right)^{+} \in W_{0}^{1, p}(\Omega)$ as test function in (3.8). With the aid of (3.3) and (3.4), we get

$$
\begin{aligned}
\left\langle A\left(u_{0}\right),\left(u_{0}-\bar{u}\right)^{+}\right\rangle & =\int_{\Omega} h_{\lambda}^{+}\left(x, u_{0}\right)\left(u_{0}-\bar{u}\right)^{+} d x \\
& =\int_{\Omega} \lambda \bar{u}^{q-1}\left(u_{0}-\bar{u}\right)^{+} d x-\int_{\Omega} f(x, \bar{u})\left(u_{0}-\bar{u}\right)^{+} d x \\
& \leq 0 .
\end{aligned}
$$

From this we see that

$$
\int_{\left\{u_{0}>\bar{u}\right\}}\left(a\left(\nabla u_{0}\right), \nabla u_{0}\right)_{\mathbb{R}^{N}} d x \leq 0
$$

meaning that, in combination with Lemma 2.7(c),

$$
\frac{c_{1}}{p-1} \int_{\left\{u_{0}>\bar{u}\right\}}\left\|\nabla u_{0}\right\|^{p} d x \leq 0
$$


We conclude that $\left|\left\{u_{0}>\bar{u}\right\}\right|_{N}=0$, thus $u_{0} \leq \bar{u}$. Recalling that $u_{0} \geq 0$ we get $u_{0} \in[0, \bar{u}]$ with $u_{0} \not \equiv 0$. Then, thanks to the definition of the truncation function in (3.4), relation (3.8) becomes

$$
A\left(u_{0}\right)=\lambda u_{0}^{q-1}-N_{f}\left(u_{0}\right)
$$

which can equivalently be written as

$$
\begin{aligned}
-\operatorname{div} a\left(\nabla u_{0}\right) & =\lambda u_{0}^{q-1}-f\left(x, u_{0}\right) & & \text { in } \Omega, \\
u_{0} & =0 & & \text { on } \partial \Omega .
\end{aligned}
$$

Since $u_{0} \in L^{\infty}(\Omega)$ (see Ladyzhenskaya-Ural'tseva [25, p. 286]) we can apply the regularity results of Lieberman [26, p. 320] to obtain that $u_{0} \in C_{0}^{1}(\bar{\Omega}) \backslash\{0\}$. Given $\varepsilon \in(0, \lambda)$, owing to hypotheses $\mathrm{H}(\mathrm{f})_{1}$ (i), (iii), we find a constant $c_{\varepsilon}>0$ such that

$$
f(x, s) \leq \varepsilon s^{q-1}+c_{\varepsilon} s^{p-1} \quad \text { for a.a. } x \in \Omega \text { and for all } 0 \leq s \leq \bar{u} .
$$

Hence, one has

$$
\operatorname{div} a\left(\nabla u_{0}(x)\right) \leq c_{\varepsilon} u_{0}(x)^{p-1} \text { a.e. in } \Omega .
$$

Then, the strong maximum principle of Pucci-Serrin [31, Theorem 2.5.1] yields $u_{0}(x)>0$ for all $x \in \Omega$. Applying the Boundary Point Lemma (see again PucciSerrin [31, Theorem 5.5.1]) gives $u_{0} \in \operatorname{int}\left(C_{0}^{1}(\bar{\Omega})_{+}\right)$.

Similarly, working with $\hat{\varphi}_{\lambda}^{-}$instead of $\hat{\varphi}_{\lambda}^{+}$, we establish the existence of a nontrivial negative solution $v_{0} \in[\underline{v}, 0] \cap\left(-\operatorname{int}\left(C_{0}^{1}(\bar{\Omega})_{+}\right)\right)$.

Our next proceeding is the proof of the existence of extremal solutions of $(P)_{\lambda}$ with $\lambda>0$ large enough as before. That means, we prove the existence of a smallest positive solution and a greatest negative solution, both of them are nontrivial and smooth.

To this end, let $S_{+}(\lambda)$ be the set containing all nontrivial positive solutions of problem $(P)_{\lambda}$. As shown in Filippakis-Kristály-Papageorgiou [19, p. 431], exploiting the monotonicity of $a(\cdot)$ (see Lemma 2.7(a)), we have that $S_{+}(\lambda)$ is downward directed, i.e., if $u_{1}, u_{2} \in S_{+}(\lambda)$, then there is an element $\tilde{u} \in S_{+}(\lambda)$ such that $\tilde{u} \leq u_{1}, \tilde{u} \leq u_{2}$. Therefore, without loss of generality, we can restrict our treatment to the set

$$
\hat{S}_{+}(\lambda)=S_{+}(\lambda) \cap[0, \bar{u}] .
$$

Likewise, if $S_{-}(\lambda)$ is the set of all nontrivial negative solutions of problem $(P)_{\lambda}$, we can focus on the set

$$
\hat{S}_{-}(\lambda)=S_{-}(\lambda) \cap[\underline{v}, 0] .
$$

By means of Proposition 3.4, we know that both sets are nonempty, i.e., $\hat{S}_{+}(\lambda) \neq$ $\emptyset$ and $\hat{S}_{-}(\lambda) \neq \emptyset$. Additionally, by truncating $f(x, \cdot)$ at $\underline{v}$ (from below) and $\bar{u}$ (from above), we may assume without loss of generality that

$$
|f(x, s)| \leq \hat{a}(x) \quad \text { for a.a. } x \in \Omega \text { and for all } s \in \mathbb{R} \text { with } \hat{a} \in L^{\infty}(\Omega)_{+}
$$

(see hypothesis $\mathrm{H}(\mathrm{f})_{1}(\mathrm{i})$ ). Using this observation and hypothesis $\mathrm{H}(\mathrm{f})_{1}(\mathrm{iii})$, we see that for a given $\varepsilon>0$ and $r \in\left(p, p^{*}\right)$, we can find $c_{7}=c_{7}(\varepsilon, r)>0$ such that

$$
f(x, s) s \leq \varepsilon|s|^{q}+c_{7}|s|^{r} \quad \text { for a.a. } x \in \Omega \text { and for all } s \in \mathbb{R} .
$$


Let $\lambda>0$ and $\varepsilon \in(0, \lambda)$. We consider the following auxiliary Dirichlet problem

$$
\begin{aligned}
-\operatorname{div} a(\nabla u) & =(\lambda-\varepsilon)|u|^{q-2} u-c_{7}|u|^{r-2} u & & \text { in } \Omega, \\
u & =0 & & \text { on } \partial \Omega .
\end{aligned}
$$

Our next result is about the uniqueness of constant sign solutions of problem (3.11).

Proposition 3.5. If hypotheses $H(a)^{\prime}$ hold and if

$$
\lambda> \begin{cases}q c_{6} \hat{\lambda}_{1}(q) & \text { if } q<p \\ 2 q c_{6} \hat{\lambda}_{1}(q) & \text { if } q=p\end{cases}
$$

is satisfied, then problem (3.11) has a unique nontrivial positive solution $u_{*} \in$ $\operatorname{int}\left(C_{0}^{1}(\bar{\Omega})_{+}\right)$and by virtue of the oddness of (3.11), $v_{*}=-u_{*} \in-\operatorname{int}\left(C_{0}^{1}(\bar{\Omega})_{+}\right)$is the unique nontrivial negative solution.

Proof. First, we establish the existence of a nontrivial positive solution for problem (3.11). To this end, we consider the $C^{1}$-functional $\psi_{\lambda}^{+}: W_{0}^{1, p}(\Omega) \rightarrow \mathbb{R}$ defined by

$$
\psi_{\lambda}^{+}(u)=\int_{\Omega} G(\nabla u) d x-\frac{\lambda-\varepsilon}{q}\left\|u^{+}\right\|_{L^{q}(\Omega)}^{q}+\frac{c_{7}}{r}\left\|u^{+}\right\|_{L^{r}(\Omega)}^{r} .
$$

Since $q \leq p<r$ and due to Corollary 2.8, we easily see that $\psi_{\lambda}^{+}$is coercive. In addition, one verifies its property to be sequentially weakly lower semicontinuous. Hence, its global minimizer exists, namely $u_{*} \in W_{0}^{1, p}(\Omega)$ satisfies

$$
\psi_{\lambda}^{+}\left(u_{*}\right)=\inf \left[\psi_{\lambda}^{+}(u): u \in W_{0}^{1, p}(\Omega)\right] .
$$

As in the proof of Proposition 3.4, the choice of $\lambda>0$ leads to

$$
\psi_{\lambda}^{+}\left(u_{*}\right)<0=\psi_{\lambda}^{+}(0)
$$

meaning that $u_{*} \neq 0$. From (3.12) we obtain $\left(\psi_{\lambda}^{+}\right)^{\prime}\left(u_{*}\right)=0$ which results in

$$
A\left(u_{*}\right)=(\lambda-\varepsilon)\left(u_{*}^{+}\right)^{q-1}-c_{7}\left(u_{*}^{+}\right)^{r-1} .
$$

Acting on (3.13) with $-u_{*}^{-} \in W_{0}^{1, p}(\Omega)$ and using Lemma 2.7, we check at once that $u_{*} \geq 0$ and $u_{*} \neq 0$. Then, (3.13) becomes

$$
A\left(u_{*}\right)=(\lambda-\varepsilon) u_{*}^{q-1}-c_{7} u_{*}^{r-1} .
$$

Hence, $u_{*}$ is a nontrivial positive solution of (3.11). Moreover, as before (see the proof of Proposition 3.4) using the nonlinear regularity theory (see [25], [26]) and the nonlinear maximum principle (see [31]), it holds $u_{*} \in \operatorname{int}\left(C_{0}^{1}(\bar{\Omega})_{+}\right)$.

It remains to show the uniqueness of this solution $u_{*}$. For this purpose, we consider the integral functional $\sigma_{+}: L^{1}(\Omega) \rightarrow \overline{\mathbb{R}}=\mathbb{R} \cup\{\infty\}$ defined by

$$
\sigma_{+}(u)= \begin{cases}\int_{\Omega} G\left(\nabla u^{\frac{1}{q}}\right) d x & \text { if } u \geq 0, u^{\frac{1}{q}} \in W_{0}^{1, p}(\Omega), \\ +\infty & \text { otherwise. }\end{cases}
$$

Let $u_{1}, u_{2} \in \operatorname{dom} \sigma_{+}$and let $u=\left(t u_{1}+(1-t) u_{2}\right)^{\frac{1}{q}}$ with $t \in[0,1]$. From Díaz-Saá [17, Lemma 1] we have

$$
\|\nabla u(x)\| \leq\left(t\left\|\nabla u_{1}(x)^{\frac{1}{q}}\right\|^{q}+(1-t)\left\|\nabla u_{2}(x)^{\frac{1}{q}}\right\|^{q}\right)^{\frac{1}{q}} \quad \text { a.e. in } \Omega,
$$


thus,

$$
\begin{aligned}
G_{0}(\|\nabla u(x)\|) & \leq G_{0}\left(\left(t\left\|\nabla u_{1}(x)^{\frac{1}{q}}\right\|^{q}+(1-t)\left\|\nabla u_{2}(x)^{\frac{1}{q}}\right\|^{q}\right)^{\frac{1}{q}}\right) \\
& \leq t G_{0}\left(\left\|\nabla u_{1}(x)^{\frac{1}{q}}\right\|\right)+(1-t) G_{0}\left(\left\|\nabla u_{2}(x)^{\frac{1}{q}}\right\|\right),
\end{aligned}
$$

thanks to hypothesis $\mathrm{H}(\mathrm{a})(\mathrm{iv})$ and the fact that $G_{0}$ is increasing. Since $G(\xi)=$ $G_{0}(\|\xi\|)$ for all $\xi \in \mathbb{R}^{N}$, it follows that

$$
G(\nabla u(x)) \leq t G\left(\nabla u_{1}(x)^{\frac{1}{q}}\right)+(1-t) G\left(\nabla u_{2}(x)^{\frac{1}{q}}\right) \quad \text { a.e. in } \Omega
$$

proving that $\sigma_{+}$is convex. Using Fatou's Lemma we infer that $\sigma_{+}$is lower semicontinuous as well.

Let $u, v \in W_{0}^{1, p}(\Omega)$ be two nontrivial positive solutions of (3.11). As done in the first part of the proof, we know that $u, v \in \operatorname{int}\left(C_{0}^{1}(\bar{\Omega})_{+}\right)$, thus $u, v \in \operatorname{dom} \sigma_{+}$. Let $h \in C_{0}^{1}(\bar{\Omega})$. For $t \in(0,1)$ sufficiently small we have $u^{q}+t h, v^{q}+t h \in \operatorname{int}\left(C_{0}^{1}(\bar{\Omega})_{+}\right)$ which implies that $\sigma_{+}$is Gateaux differentiable at $u^{q}$ and $v^{q}$ in the direction $h$. Moreover, via the chain rule, we obtain

$$
\begin{aligned}
\sigma_{+}^{\prime}\left(u^{q}\right)(h) & =\frac{1}{q} \int_{\Omega} \frac{-\operatorname{div} a(\nabla u)}{u^{q-1}} h d x, \\
\sigma_{+}^{\prime}\left(v^{q}\right)(h) & =\frac{1}{q} \int_{\Omega} \frac{-\operatorname{div} a(\nabla v)}{v^{q-1}} h d x .
\end{aligned}
$$

Since $\sigma_{+}$is convex, it is clear that $\sigma_{+}^{\prime}$ is monotone. Therefore, using (3.14) and (3.15), it holds

$$
\begin{aligned}
0 & \leq\left\langle\sigma_{+}^{\prime}\left(u^{q}\right)-\sigma_{+}^{\prime}\left(v^{q}\right), u^{q}-v^{q}\right\rangle_{L^{1}(\Omega)} \\
& =\frac{1}{q} \int_{\Omega}\left(\frac{-\operatorname{div} a(\nabla u)}{u^{q-1}}+\frac{\operatorname{div} a(\nabla v)}{v^{q-1}}\right)\left(u^{q}-v^{q}\right) d x \\
& =\frac{1}{q} \int_{\Omega}\left(\frac{(\lambda-\varepsilon) u^{q-1}-c_{7} u^{r-1}}{u^{q-1}}-\frac{(\lambda-\varepsilon) v^{q-1}-c_{7} v^{r-1}}{v^{q-1}}\right)\left(u^{q}-v^{q}\right) d x \\
& =\frac{c_{7}}{q} \int_{\Omega}\left(v^{r-q}-u^{r-q}\right)\left(u^{q}-v^{q}\right) d x .
\end{aligned}
$$

Since $s \mapsto s^{r-q}$ is strictly increasing in $(0, \infty)$, it follows that $u=v$, hence $u_{*} \in$ $\operatorname{int}\left(C_{0}^{1}(\bar{\Omega})_{+}\right)$is the unique nontrivial positive solution of (3.11).

Evidently the oddness of (3.11) implies that $v_{*}=-u_{*} \in-\operatorname{int}\left(C_{0}^{1}(\bar{\Omega})_{+}\right)$is the unique nontrivial negative solution of (3.11).

Now, these two solutions $u_{*} \in \operatorname{int}\left(C_{0}^{1}(\bar{\Omega})_{+}\right)$and $v_{*}=-\operatorname{int}\left(C_{0}^{1}(\bar{\Omega})_{+}\right)$of the auxiliary problem (3.11) lead to the existence of extremal nontrivial constant sign solutions for problem $(P)_{\lambda}$ when $\lambda>0$ is sufficiently large. We obtain the following result.

Proposition 3.6. Let hypotheses $H(a)^{\prime}$ and $H(f)_{1}$ hold and suppose

$$
\lambda> \begin{cases}q c_{6} \hat{\lambda}_{1}(q) & \text { if } q<p, \\ 2 q c_{6} \hat{\lambda}_{1}(q) & \text { if } q=p .\end{cases}
$$

Then problem $(P)_{\lambda}$ has a smallest nontrivial positive solution $u_{+} \in \operatorname{int}\left(C_{0}^{1}(\bar{\Omega})_{+}\right)$ and a greatest nontrivial negative solution $v_{-} \in-\operatorname{int}\left(C_{0}^{1}(\bar{\Omega})_{+}\right)$. 
Proof. As we already noted, it suffices to establish the existence of a smallest nontrivial element in $\hat{S}_{+}(\lambda)=S_{+}(\lambda) \cap[0, \bar{u}] \subseteq \operatorname{int}\left(C_{0}^{1}(\bar{\Omega})_{+}\right)$and the existence of a greatest nontrivial negative solution in $\hat{S}_{-}(\lambda)=S_{-}(\lambda) \cap[\underline{v}, 0] \subseteq-\operatorname{int}\left(C_{0}^{1}(\bar{\Omega})_{+}\right)$.

Claim: $u_{*} \leq u$ for all $u \in \hat{S}_{+}(\lambda)$.

Let $\tilde{u} \in \hat{S}_{*}(\lambda)$ and consider the Carathéodory function

$$
\gamma_{\lambda}^{+}(x, s)= \begin{cases}0 & \text { if } s<0, \\ (\lambda-\varepsilon) s^{q-1}-c_{7} s^{r-1} & \text { if } 0 \leq s \leq \tilde{u}(x), \\ (\lambda-\varepsilon) \tilde{u}(x)^{q-1}-c_{7} \tilde{u}(x)^{r-1} & \text { if } \tilde{u}(x)<s .\end{cases}
$$

We set $\Gamma_{\lambda}^{+}(x, s)=\int_{0}^{s} \gamma_{\lambda}^{+}(x, t) d t$ and consider the $C^{1}$-functional $\hat{\psi}_{\lambda}^{+}: W_{0}^{1, p}(\Omega) \rightarrow \mathbb{R}$ defined by

$$
\hat{\psi}_{\lambda}^{+}(u)=\int_{\Omega} G(\nabla u) d x-\int_{\Omega} \Gamma_{\lambda}^{+}(x, u) d x .
$$

Thanks to (3.16) it is obvious that $\hat{\psi}_{\lambda}^{+}$is coercive and it is also sequentially weakly lower semicontinuous. Thus, we find $\hat{u}_{*} \in W_{0}^{1, p}(\Omega)$ such that

$$
\hat{\psi}_{\lambda}^{+}\left(\hat{u}_{*}\right)=\inf \left[\hat{\psi}_{\lambda}^{+}(u): u \in W_{0}^{1, p}(\Omega)\right] .
$$

As before (see the proof of Proposition 3.4 and recall the choice of $\lambda>0$ ), we have

$$
\hat{\psi}_{\lambda}^{+}\left(\hat{u}_{*}\right)<0=\hat{\psi}_{\lambda}^{+}(0)
$$

hence $\hat{u}_{*} \neq 0$. From (3.17) we have

$$
A\left(\hat{u}_{*}\right)=N_{\gamma_{\lambda}^{+}}\left(\hat{u}_{*}\right) .
$$

Applying again $-\hat{u}_{*}^{-} \in W_{0}^{1, p}(\Omega)$ as test function to (3.18), it follows from Lemma $2.7(\mathrm{c})$ that $\hat{u}_{*} \geq 0, \hat{u}_{*} \neq 0$. Furthermore, we act on (3.18) with $\left(\hat{u}_{*}-\tilde{u}\right)^{+} \in$ $W_{0}^{1, p}(\Omega)$. Then, from (3.10), (3.16) and the fact that $\tilde{u} \in \hat{S}_{+}(\lambda)$, it follows

$$
\begin{aligned}
\left\langle A\left(\hat{u}_{*}\right),\left(\hat{u}_{*}-\tilde{u}\right)^{+}\right\rangle & =\int_{\Omega} \gamma_{\lambda}^{+}\left(x, \hat{u}_{*}\right)\left(\hat{u}_{*}-\tilde{u}\right)^{+} d x \\
& =\int_{\Omega}\left((\lambda-\varepsilon) \tilde{u}^{q-1}-c_{7} \tilde{u}^{r-1}\right)\left(\hat{u}_{*}-\tilde{u}\right)^{+} d x \\
& \leq \int_{\Omega}\left(\lambda \tilde{u}^{q-1}-f(u, \tilde{u})\right)\left(\hat{u}_{*}-\tilde{u}\right)^{+} d x \\
& =\left\langle A(\tilde{u}),\left(\hat{u}_{*}-\tilde{u}\right)^{+}\right\rangle .
\end{aligned}
$$

We deduce

$$
\int_{\left\{\hat{u}_{*}>\tilde{u}\right\}}\left(a\left(\nabla \hat{u}_{*}\right)-a(\nabla \tilde{u}), \nabla \hat{u}_{*}-\nabla \tilde{u}\right)_{\mathbb{R}^{N}} d x \leq 0,
$$

which implies that $\left|\left\{\hat{u}_{*}>\tilde{u}\right\}\right|_{N}=0$ due to Lemma 2.7(a). Hence

$$
0 \neq \hat{u}_{*} \in[0, \tilde{u}]=\left\{u \in W_{0}^{1, p}(\Omega): 0 \leq u(x) \leq \tilde{u}(x) \text { a.e. in } \Omega\right\} .
$$

By virtue of (3.16) and (3.18), we see that $\hat{u}_{*}$ is a nontrivial positive solution of (3.11), hence $\hat{u}_{*}=u_{*} \in \operatorname{int}\left(C_{0}^{1}(\bar{\Omega})_{+}\right)$(see Proposition 3.5). Therefore $u_{*} \leq \tilde{u}$ and since $\tilde{u} \in \hat{S}_{+}(\lambda)$ is arbitrary, we have proved the claim. 
Let $C \subseteq \hat{S}_{+}(\lambda)$ be a chain, i.e., a totally ordered subset of $\hat{S}_{+}(\lambda)$. According to Dunford-Schwartz [18, p. 336], there exists a sequence $\left(u_{n}\right)_{n \geq 1} \subseteq \hat{S}_{+}(\lambda)$ such that

$$
\inf C=\inf _{n \geq 1} u_{n}
$$

We have

$$
A\left(u_{n}\right)=\lambda u_{n}^{q-1}-N_{f}\left(u_{n}\right), \quad u_{*} \leq u_{n} \leq \bar{u} \quad \text { for all } n \geq 1 .
$$

Therefore, $\left(u_{n}\right)_{n \geq 1} \subseteq W_{0}^{1, p}(\Omega)$ is bounded and we may assume that

$$
u_{n} \rightarrow u \text { in } W_{0}^{1, p}(\Omega) \quad \text { and } \quad u_{n} \rightarrow u \text { in } L^{p}(\Omega) .
$$

Acting on (3.19) with the test function $u_{n}-u \in W_{0}^{1, p}(\Omega)$ and passing to the limit as $n \rightarrow \infty$ combined with (3.20), we obtain

$$
\lim _{n \rightarrow \infty}\left\langle A\left(u_{n}\right), u_{n}-u\right\rangle=0 .
$$

By means of Proposition 2.11, we know that $A$ fulfills the $\left(\mathrm{S}_{+}\right)$-property which in view of (3.21) and the weak convergence in $W_{0}^{1, p}(\Omega)$ gives $u_{n} \rightarrow u$ in $W_{0}^{1, p}(\Omega)$. Then, we directly obtain from (3.19) that

$$
A(u)=\lambda u^{q-1}-N_{f}(u), \quad u_{*} \leq u \leq \bar{u}
$$

meaning that $u \in \hat{S}_{+}(\lambda)$ and $u=\inf C$. Since $C$ is an arbitrary chain in $\hat{S}_{+}(\lambda)$, the Kuratowski-Zorn Lemma implies that $\hat{S}_{+}(\lambda)$ has a minimal element $u_{+} \in \hat{S}_{+}(\lambda)$. Since $\hat{S}_{+}(\lambda)$ is downward directed, we conclude that $u_{+}$is the smallest nontrivial positive solution of $(P)_{\lambda}$.

Similarly, working with the set $\hat{S}_{-}(\lambda)$ and using again the Kuratowski-Zorn Lemma, we obtain that $v_{-} \in-\operatorname{int}\left(C_{0}^{1}(\bar{\Omega})_{+}\right)$is the greatest nontrivial negative solution of $(P)_{\lambda}$.

Finally, we will prove the existence of a sign-changing solution $y_{0}$ of $(P)_{\lambda}$ lying between these two extremal nontrivial constant sign solutions obtained in the last proposition.

Proposition 3.7. If hypotheses $H(a)^{\prime}$ and $H(f)_{1}$ hold and if

$$
\lambda> \begin{cases}q c_{6} \hat{\lambda}_{2}(q) & \text { if } q<p, \\ 2 q c_{6} \hat{\lambda}_{2}(q) & \text { if } q=p,\end{cases}
$$

is satisfied, then problem $(P)_{\lambda}$ has a nodal solution $y_{0} \in C_{0}^{1}(\bar{\Omega})$.

Proof. Let $u_{+} \in \operatorname{int}\left(C_{0}^{1}(\bar{\Omega})_{+}\right)$and $v_{-} \in-\operatorname{int}\left(C_{0}^{1}(\bar{\Omega})_{+}\right)$be the two extremal nontrivial constant sign solutions of $(P)_{\lambda}$ produced in Proposition 3.6. We introduce the following truncation function of the reaction of problem $(P)_{\lambda}$

$$
h_{\lambda}(x, s)= \begin{cases}\lambda\left|v_{-}(x)\right|^{q-2} v_{-}(x)-f\left(x, v_{-}(x)\right) & \text { if } s<v_{-}(x), \\ \lambda|s|^{q-2} s-f(x, s) & \text { if } v_{-}(x) \leq s \leq u_{+}(x), \\ \lambda u_{+}(x)^{q-1}-f\left(x, u_{+}(x)\right) & \text { if } u_{+}(x)<s .\end{cases}
$$

Of course, $h: \Omega \times \mathbb{R} \rightarrow \mathbb{R}$ is a Carathéodory function. In addition, we need the positive and negative truncations of $h_{\lambda}(x, \cdot)$, namely the Carathéodory functions $h_{\lambda}^{ \pm}(x, s)=h_{\lambda}\left(x, \pm s^{ \pm}\right)$. Let $H_{\lambda}(x, s)=\int_{0}^{s} h_{\lambda}(x, t) d t$ and $H_{\lambda}^{ \pm}(x, s)=$ 
$\int_{0}^{s} h_{\lambda}^{ \pm}(x, t) d t$. We consider the $C^{1}$-functionals $\beta_{\lambda}, \beta_{\lambda}^{ \pm}: W_{0}^{1, p}(\Omega) \rightarrow \mathbb{R}$ defined by

$$
\begin{aligned}
& \beta_{\lambda}(u)=\int_{\Omega} G(\nabla u) d x-\int_{\Omega} H_{\lambda}(x, u) d x, \\
& \beta_{\lambda}^{ \pm}(u)=\int_{\Omega} G(\nabla u) d x-\int_{\Omega} H_{\lambda}^{ \pm}(x, u) d x .
\end{aligned}
$$

\section{Claim 1:}

$$
\begin{aligned}
& K_{\beta_{\lambda}} \subseteq\left[v_{-}, u_{+}\right]=\left\{u \in W_{0}^{1, p}(\Omega): v_{-}(x) \leq u(x) \leq u_{+}(x) \text { a.e. in } \Omega\right\}, \\
& K_{\beta_{\lambda}^{+}}=\left\{0, u_{+}\right\}, \quad K_{\beta_{\lambda}^{-}}=\left\{v_{-}, 0\right\} .
\end{aligned}
$$

Let $u \in K_{\beta_{\lambda}}$. Then

$$
A(u)=N_{h_{\lambda}}(u)
$$

Taking $\left(u-u_{+}\right)^{+} \in W_{0}^{1, p}(\Omega)$ as test function in (3.23) we derive thanks to (3.22)

$$
\begin{aligned}
\left\langle A(u),\left(u-u_{+}\right)^{+}\right\rangle & =\int_{\Omega} h_{\lambda}(x, u)\left(u-u_{+}\right)^{+} d x \\
& =\int_{\Omega}\left[\lambda u_{+}^{q-1}-f\left(x, u_{+}\right)\right]\left(u-u_{+}\right)^{+} d x \\
& =\left\langle A\left(u_{+}\right),\left(u-u_{+}\right)^{+}\right\rangle,
\end{aligned}
$$

hence,

$$
\int_{\left\{u>u_{+}\right\}}\left(a(\nabla u)-a\left(\nabla u_{+}\right), \nabla u-\nabla u_{+}\right)_{\mathbb{R}^{N}} d x=0 .
$$

This gives $\left|\left\{u>u_{+}\right\}\right|_{N}=0$ (see Lemma 2.7(a)), thus $u \leq u_{+}$.

Similarly, acting on (3.23) with $\left(v_{-}-u\right)^{+} \in W_{0}^{1, p}(\Omega)$ we obtain $v_{-} \leq u$. Hence

$$
K_{\beta_{\lambda}} \subseteq\left[v_{-}, u_{+}\right]
$$

In the same way, we show that

$$
\begin{aligned}
& K_{\beta_{\lambda}^{+}} \subseteq\left[0, u_{+}\right]=\left\{u \in W_{0}^{1, p}(\Omega): 0 \leq u(x) \leq u_{+}(x) \text { a.e. in } \Omega\right\}, \\
& K_{\beta_{\lambda}^{-}} \subseteq\left[v_{-}, 0\right]=\left\{u \in W_{0}^{1, p}(\Omega): v_{-}(x) \leq u(x) \leq 0 \text { a.e. in } \Omega\right\} .
\end{aligned}
$$

Since $u_{+}$and $v_{-}$are the extremal constant sign solutions of $(P)_{\lambda}$ and due to the fact that the positive and negative solutions of $(P)_{\lambda}$ are exactly the critical points of $\beta_{\lambda}^{+}$and $\beta_{\lambda}^{-}$, respectively, we infer that

$$
K_{\beta_{\lambda}^{+}}=\left\{0, u_{+}\right\} \quad \text { and } \quad K_{\beta_{\lambda}^{-}}=\left\{v_{-}, 0\right\} .
$$

This proves Claim 1 .

Claim 2: $u_{+} \in \operatorname{int}\left(C_{0}^{1}(\bar{\Omega})_{+}\right)$and $v_{-} \in-\operatorname{int}\left(C_{0}^{1}(\bar{\Omega})_{+}\right)$are local minimizers of $\beta_{\lambda}$.

Clearly, the functional $\beta_{\lambda}^{+}$is coercive and sequentially weakly lower semicontinuous. Then we find $\hat{u} \in W_{0}^{1, p}(\Omega)$ such that

$$
\beta_{\lambda}^{+}(\hat{u})=\inf \left\{\beta_{\lambda}^{+}(u): u \in W_{0}^{1, p}(\Omega)\right\} .
$$

As in the proof of Proposition 3.4, we can show that $\beta_{\lambda}^{+}(\hat{u})<0=\beta_{\lambda}^{+}(0)$, hence $\hat{u} \neq 0$. Therefore $\hat{u}=u_{+} \in \operatorname{int}\left(C_{0}^{1}(\bar{\Omega})_{+}\right)$(see Claim 1). Note that $\left.\beta_{\lambda}\right|_{C_{0}^{1}(\bar{\Omega})_{+}}=$ 
$\left.\beta_{\lambda}^{+}\right|_{C_{0}^{1}(\bar{\Omega})_{+}}$. Then, $u_{+} \in \operatorname{int}\left(C_{0}^{1}(\bar{\Omega})_{+}\right)$is a local $C_{0}^{1}(\bar{\Omega})$-minimizer of $\beta_{\lambda}$ and by virtue of Proposition $2.10 u_{+}$is a local $W_{0}^{1, p}(\Omega)$-minimizer of $\beta_{\lambda}$. Similarly, using $\beta_{\lambda}^{-}$instead of $\beta_{\lambda}^{+}$, we obtain the assertion for $v_{-} \in-\operatorname{int}\left(C_{0}^{1}(\bar{\Omega})_{+}\right)$. This proves Claim 2.

Without loss of generality, we may assume that $\beta_{\lambda}\left(v_{-}\right) \leq \beta_{\lambda}\left(u_{+}\right)$(the analysis is similar if the opposite inequality holds). Furthermore, we may assume that $u_{+}$is an isolated element of $K_{\beta_{\lambda}}$ (otherwise we have a whole sequence of distinct nontrivial solutions of $\left.(P)_{\lambda}\right)$. Then, there exists $\rho \in(0,1)$ such that $\left\|v_{-}-u_{+}\right\|_{W_{0}^{1, p}(\Omega)}>\rho$ and

$$
\beta_{\lambda}\left(v_{-}\right) \leq \beta_{\lambda}\left(u_{+}\right)<\inf \left[\beta_{\lambda}(u):\left\|u-u_{+}\right\|_{W_{0}^{1, p}(\Omega)}=\rho\right]=\eta_{\rho}^{\lambda}
$$

(see, for example, Aizicovici-Papageorgiou-Staicu [1, Proof of Proposition 29]). Since $\beta_{\lambda}$ is coercive it satisfies the PS-condition (see Proposition 2.13). This fact and (3.24) permit the application of the Mountain Pass Theorem (Theorem 2.2), which ensures the existence of an element $y_{0} \in W_{0}^{1, p}(\Omega)$ such that

$$
y_{0} \in K_{\beta_{\lambda}} \subseteq\left[v_{-}, u_{+}\right] \text {(see Claim 1) and } \eta_{\rho}^{\lambda} \leq \beta_{\lambda}\left(y_{0}\right) .
$$

From (3.25) it follows that $y_{0}$ is a solution of $(P)_{\lambda}$ (see (3.22)) and $y_{0} \notin\left\{v_{-}, u_{+}\right\}$ (see also (3.25)). The nonlinear regularity theory implies that $y_{0} \in C_{0}^{1}(\bar{\Omega})$. Since $y_{0} \in\left[v_{-}, u_{+}\right] \backslash\left\{v_{-}, u_{+}\right\}$, the extremality of $v_{-}$and $u_{+}$implies that $y_{0}$ is nodal provided $y_{0} \neq 0$.

Thanks to Theorem 2.2 we also have that

$$
\beta_{\lambda}\left(y_{0}\right)=\inf _{\gamma \in \Gamma} \max _{0 \leq t \leq 1} \beta_{\lambda}(\gamma(t)),
$$

where $\Gamma=\left\{\gamma \in C\left([0,1], W_{0}^{1, p}(\Omega)\right): \gamma(0)=v_{-}, \gamma(1)=u_{+}\right\}$. In order to show the nontriviality of $y_{0}$ it suffices to produce a path $\gamma_{*} \in \Gamma$ such that $\beta_{\lambda}\left(\gamma_{*}(t)\right) \neq 0$ for all $t \in[0,1]$ (see (3.26)).

By means of hypothesis $\mathrm{H}(\mathrm{f})_{1}(\mathrm{iii})$, given $\varepsilon>0$, we find $\delta=\delta(\varepsilon)>0$ such that

$$
|f(x, s)| \leq \varepsilon|s|^{q-1} \quad \text { for a.a. } x \in \Omega \text { and for all }|s| \leq \delta,
$$

which implies that

$$
F(x, s) \leq \frac{\varepsilon}{q}|s|^{q} \quad \text { for a.a. } x \in \Omega \text { and for all }|s| \leq \delta .
$$

Recall (see Section 2) that $\partial B_{1}^{L^{q}}=\left\{u \in L^{q}(\Omega):\|u\|_{L^{q}(\Omega)}=1\right\}$ and $M_{q}=W_{0}^{1, q}(\Omega) \cap$ $\partial B_{1}^{L^{q}}$. Defining $M_{q}^{c}=M_{q} \cap C_{0}^{1}(\bar{\Omega})$, we endow $M_{q}$ with the relative $W_{0}^{1, p}(\Omega)$ topology and $M_{q}^{c}$ with the relative $C_{0}^{1}(\bar{\Omega})$-topology. Evidently, $M_{q}^{c}$ is dense in $M_{q}$ which implies that $C\left([-1,1], M_{q}^{c}\right)$ is dense in $C\left([-1,1], M_{q}\right)$ as well. We consider the following two sets of continuous paths

$$
\begin{aligned}
& \hat{\Gamma}(q)=\left\{\hat{\gamma} \in C\left([-1,1], M_{q}\right): \hat{\gamma}(-1)=-\hat{u}_{1}(q), \hat{\gamma}(1)=\hat{u}_{1}(q)\right\},(\text { cf. Section } 2), \\
& \hat{\Gamma}_{c}(q)=\left\{\hat{\gamma} \in C\left([-1,1], M_{q}^{c}\right): \hat{\gamma}(-1)=-\hat{u}_{1}(q), \hat{\gamma}(1)=\hat{u}_{1}(q)\right\}
\end{aligned}
$$

Note that $\hat{\Gamma}_{c}(q)$ is dense in $\hat{\Gamma}(q)$. Taking into account the variational characterization of the second eigenvalue $\hat{\lambda}_{2}(q)$ (see Proposition 2.12), we find $\hat{\gamma} \in \hat{\Gamma}(q)$ such that

$$
\max _{-1 \leq t \leq 1}\|\nabla \hat{\gamma}(t)\|_{L^{q}(\Omega)}^{q}<\hat{\lambda}_{2}(q)+\frac{\delta}{2}
$$


The density of $\hat{\Gamma}_{c}(q)$ in $\hat{\Gamma}(q)$ implies that for a given $\varepsilon>0$ there exists $\hat{\gamma}_{0} \in \hat{\Gamma}_{c}(q)$ such that

$$
\max _{-1 \leq t \leq 1}\left\|\hat{\gamma}(t)-\hat{\gamma}_{0}(t)\right\|_{W_{0}^{1, q}(\Omega)}<\varepsilon
$$

from which we derive that

$$
\max _{-1 \leq t \leq 1}\left\|\nabla \hat{\gamma}(t)-\nabla \hat{\gamma}_{0}(t)\right\|_{L^{q}(\Omega)}<\varepsilon
$$

Combining (3.28) and (3.29), there holds, for every $t \in[-1,1]$,

$$
\begin{aligned}
\left\|\nabla \hat{\gamma}_{0}(t)\right\|_{L^{q}(\Omega)} & \leq\left\|\nabla \hat{\gamma}_{0}(t)-\nabla \hat{\gamma}(t)\right\|_{L^{q}(\Omega)}+\|\nabla \hat{\gamma}(t)\|_{L^{q}(\Omega)} \\
& <\varepsilon+\left(\hat{\lambda}_{2}(q)+\frac{\delta}{2}\right)^{\frac{1}{q}}
\end{aligned}
$$

Choosing $0<\varepsilon<\left(\hat{\lambda}_{2}(q)+\delta\right)^{\frac{1}{q}}-\left(\hat{\lambda}_{2}(q)+\frac{\delta}{2}\right)^{\frac{1}{q}}$, we obtain

$$
\left\|\nabla \hat{\gamma}_{0}(t)\right\|_{L^{q}(\Omega)}<\left(\hat{\lambda}_{2}(q)+\delta\right)^{\frac{1}{q}} \quad \text { for all } t \in[-1,1]
$$

meaning that

$$
\max _{-1 \leq t \leq 1}\left\|\nabla \hat{\gamma}_{0}(t)\right\|_{L^{q}(\Omega)}^{q}<\hat{\lambda}_{2}(q)+\delta .
$$

Since $\hat{\gamma}_{0}([-1,1]) \subseteq C_{0}^{1}(\bar{\Omega})$ is compact and due to $u_{+} \in \operatorname{int}\left(C_{0}^{1}(\bar{\Omega})_{+}\right), v_{-} \in-\operatorname{int}\left(C_{0}^{1}(\bar{\Omega})_{+}\right)$, we can find $\eta \in(0,1)$ such that

$$
|\eta u(x)| \leq \delta
$$

for all $x \in \bar{\Omega}$, all $u \in \hat{\gamma}_{0}([-1,1])$, and $\eta u \in\left[v_{-}, u_{+}\right]$for all $u \in \hat{\gamma}_{0}([-1,1])$.

First let us suppose that $q<p$. Then, thanks to Corollary $2.8,\|u\|_{L^{q}(\Omega)}=1$, (3.27), (3.30), (3.31), and $u \in \hat{\gamma}_{0}([-1,1])$, it follows

$$
\begin{aligned}
\beta_{\lambda}(\eta u) & =\int_{\Omega} G(\nabla(\eta u)) d x-\int_{\Omega} H_{\lambda}(x, \eta u) d x \\
& \leq c_{5}\left(\eta^{q}\|\nabla u\|_{L^{q}(\Omega)}^{q}+\eta^{p}\|\nabla u\|_{L^{p}(\Omega)}^{p}\right)-\frac{\lambda \eta^{q}}{q}+\int_{\Omega} F(x, \eta u) d x \\
& \leq c_{5}\left(\eta^{q}\|\nabla u\|_{L^{q}(\Omega)}^{q}+\eta^{p}\|\nabla u\|_{L^{p}(\Omega)}^{p}\right)-\frac{\lambda \eta^{q}}{q}+\frac{\varepsilon}{q} \eta^{q} \\
& \leq \eta^{q}\left[c_{5}\left(\hat{\lambda}_{2}(q)+\delta\right)-\frac{\lambda-\varepsilon}{q}\right]+c_{5} \eta^{p}\|\nabla u\|_{L^{p}(\Omega)}^{p} .
\end{aligned}
$$

Since $\hat{\gamma}_{0}([-1,1]) \subseteq C_{0}^{1}(\bar{\Omega})$ is compact, there exists $\varepsilon^{*}>0$ such that

$$
\|\nabla u\|_{L^{p}(\Omega)}^{p} \leq \varepsilon^{*} \quad \text { for all } u \in \hat{\gamma}_{0}([-1,1]) .
$$

We choose $\varepsilon>0$ and $\delta \in(0, \varepsilon)$ such that $q c_{5} \delta+\varepsilon<\lambda-q c_{5} \hat{\lambda}_{2}(q)$. Then, due to (3.32), it holds

$$
\beta_{\lambda}(\eta u) \leq-\eta^{q} \hat{\varepsilon}^{*}+\eta^{p} \varepsilon^{*} \quad \text { for some } \hat{\varepsilon}^{*}>0 .
$$

Because $q<p$, by choosing $\eta \in(0,1)$ even smaller if necessary, we get

$$
\beta_{\lambda}(\eta u)<0 \text { for all } u \in \hat{\gamma}_{0}([-1,1]) .
$$

If $q=p$, the above argument works for $\lambda>2 q c_{5} \hat{\lambda}_{2}(q)$. 
Setting $\gamma_{0}=\eta \hat{\gamma}_{0}$, we see that $\gamma_{0}$ is a continuous path in $W_{0}^{1, p}(\Omega)\left(\right.$ since $\left.\gamma_{0}([-1,1]) \subseteq C_{0}^{1}(\bar{\Omega})\right)$, which connects $-\eta \hat{u}_{1}(q)$ and $\eta \hat{u}_{1}(q)$ satisfying

$$
\left.\beta_{\lambda}\right|_{\gamma_{0}}<0
$$

Recalling that $K_{\beta_{\lambda}^{+}}=\left\{0, u_{+}\right\}$(see Claim 1), we have

$$
\beta_{\lambda}^{+}\left(u_{+}\right)=\inf _{u \in W_{0}^{1, p}(\Omega)} \beta_{\lambda}^{+}(u)<0=\beta_{\lambda}^{+}(0)
$$

(cf. the proof of Claim 2). Invoking the Second Deformation Lemma (see Lemma 2.3) with $a=\beta_{\lambda}^{+}\left(u_{+}\right)<0=\beta_{\lambda}^{+}(0)=b$, we find a homotopy $h:[0,1] \times$ $\left(\left(\beta_{\lambda}^{+}\right)^{0} \backslash\{0\}\right) \rightarrow\left(\beta_{\lambda}^{+}\right)^{0}$ such that

$$
h\left(1,\left(\beta_{\lambda}^{+}\right)^{0} \backslash\{0\}\right)=\left\{u_{+}\right\}
$$

(see (3.34) and Claim 1) and

$$
\beta_{\lambda}^{+}(h(t, u)) \leq \beta_{\lambda}^{+}(u) \text { for all } t \in[0,1] \text { and all } u \in\left(\beta_{\lambda}^{+}\right)^{0} \backslash\{0\} .
$$

We set $\gamma_{+}(t)=\left(h\left(t, \eta \hat{u}_{1}(q)\right)\right)^{+}$for all $t \in[0,1]$ (see (3.33)). Obviously, $\gamma_{+}$is a continuous path in $W_{0}^{1, p}(\Omega)$ and we have

$$
\begin{aligned}
& \gamma_{+}(0)=\left(h\left(0, \eta \hat{u}_{1}(q)\right)\right)^{+}=\eta \hat{u}_{1}(q) \\
& \gamma_{+}(1)=\left(h\left(1, \eta \hat{u}_{1}(q)\right)^{+}=u_{+},\right.
\end{aligned}
$$

due to (3.35). Furthermore, (3.36) and (3.33) imply that

$$
\beta_{\lambda}^{+}\left(\gamma_{+}(t)\right) \leq \beta_{\lambda}^{+}\left(\eta \hat{u}_{1}(q)\right)<0 \quad \text { for all } t \in[0,1] .
$$

Hence, $\left.\beta_{\lambda}^{+}\right|_{\gamma_{+}}<0$. If $W_{+}^{p}=\left\{u \in W_{0}^{1, p}(\Omega): u(x) \geq 0\right.$ a.e. in $\left.\Omega\right\}$, then

$$
\left.\beta_{\lambda}\right|_{W_{+}^{p}}=\left.\beta_{\lambda}^{+}\right|_{W_{+}^{p}} \text { and } \operatorname{im} \gamma_{+} \subseteq W_{+}^{p},
$$

which yields

$$
\left.\beta_{\lambda}\right|_{\gamma_{+}}<0
$$

In a similar fashion, we produce another continuous path $\gamma_{-}$in $W_{0}^{1, p}(\Omega)$ which connects $-\eta \hat{u}_{1}(q)$ and $v_{-}$. Furthermore, we have again

$$
\left.\beta_{\lambda}\right|_{\gamma_{-}}<0
$$

We concatenate $\gamma_{-}, \gamma_{0}$, and $\gamma_{+}$to obtain a continuous path $\gamma_{*} \in \Gamma$ such that

$$
\left.\beta_{\lambda}\right|_{\gamma_{*}}<0
$$

due to (3.33), (3.37), and (3.38). This implies that $y_{0} \neq 0$, so $y_{0} \in C_{0}^{1}(\bar{\Omega})$ is a nodal solution of $(P)_{\lambda}$.

Now, we can state the following multiplicity result for problem $(P)_{\lambda}$.

Theorem 3.8. If hypotheses $H(a)_{1}$ and $H(f)_{1}$ hold and assume

$$
\lambda> \begin{cases}q c_{6} \hat{\lambda}_{2}(q) & \text { if } q<p, \\ 2 q c_{6} \hat{\lambda}_{2}(q) & \text { if } q=p,\end{cases}
$$

then problem $(P)_{\lambda}$ has at least three nontrivial solutions, namely $u_{0} \in \operatorname{int}\left(C_{0}^{1}(\bar{\Omega})_{+}\right), v_{0} \in$ $-\operatorname{int}\left(C_{0}^{1}(\bar{\Omega})_{+}\right)$and a nodal solution $y_{0} \in\left[v_{0}, u_{0}\right] \cap C_{0}^{1}(\bar{\Omega})$. Furthermore, $(P)_{\lambda}$ has 
a smallest nontrivial positive solution $u_{+} \in \operatorname{int}\left(C_{0}^{1}(\bar{\Omega})_{+}\right)$and a greatest nontrivial negative solution $v_{-} \in-\operatorname{int}\left(C_{0}^{1}(\bar{\Omega})_{+}\right)$.

Remark 3.9. Note that if $q=p$ and $a(\xi)=\|\xi\|^{p-2} \xi$ for all $\xi \in \mathbb{R}^{N}$, then $c_{6}=\frac{1}{2 q}$ and consequently $2 q c_{6} \hat{\lambda}_{2}(q)=\hat{\lambda}_{2}(q)$. Therefore, Theorem 3.8 recovers the multiplicity results of [2], [3], [33], [34] (where $p=2$ ) and [5], [8], [29] (where $p>1$ ). In fact, even in this special case, our result is more general than those in the aforementioned works. Indeed, in the semilinear works (i.e., $p=2$ ), the perturbation $f(x, s)=f(s)$ is either $C^{1}$ (see [2], [3], [33]) or Lipschitz continuous (see [34]). In the quasilinear works (i.e., $p>1$, see [5], [29]), the perturbation $f(x, s)$ is assumed to have strictly subcritical growth in the variable $s \in \mathbb{R}$. In contrast here no growth restriction is imposed on $f(x, \cdot)$ (see hypothesis $\mathrm{H}(\mathrm{f})_{1}(\mathrm{i})$ ).

\section{The $(p, 2)$-EQUation}

In this section we consider the special case $a(\xi)=\|\xi\|^{p-2} \xi+\xi$ for all $\xi \in \mathbb{R}^{N}$ with $2<p<\infty$ and $q=2$. Then, problem $(P)_{\lambda}$ becomes

$$
\begin{aligned}
-\Delta_{p} u-\Delta u & =\lambda u-f(x, u) & & \text { in } \Omega, \\
u & =0 & & \text { on } \partial \Omega .
\end{aligned}
$$

Applying Morse theory (critical groups), we are going to show that for all $\lambda>0$ sufficiently large not being in the spectrum of $\left(-\Delta, H_{0}^{1}(\Omega)\right)$, problem $(Q)_{\lambda}$ has at least four nontrivial smooth solutions whereby two of them have constant sign and the other ones have changing sign. To do so, we need to strengthen our hypotheses on the perturbation $f: \Omega \times \mathbb{R} \rightarrow \mathbb{R}$ in the following way.

$\mathrm{H}(\mathrm{f})_{2}: f: \Omega \times \mathbb{R} \rightarrow \mathbb{R}$ is a measurable function such that $f(x, \cdot) \in C^{1}(\mathbb{R})$ for a.a. $x \in \Omega$ and

(i) $\left|f_{s}^{\prime}(x, s)\right| \leq c|s|^{r-2}$ for a.a. $x \in \Omega$, for all $s \in \mathbb{R}$, with $c>0$, and $p<r<p^{*}$

(ii) $\lim _{s \rightarrow \pm \infty} \frac{f(x, s)}{|s|^{p-2} s}=+\infty$ uniformly for a.a. $x \in \Omega$;

(iii) $f_{s}^{\prime}(x, 0)=\lim _{s \rightarrow 0} \frac{f(x, s)}{s}=0$ uniformly for a.a. $x \in \Omega$.

Remark 4.1. These hypotheses are similar to those in $\mathrm{H}(\mathrm{f})_{1}$. However, we now require that $f(x, \cdot)$ is differentiable for a.a. $x \in \Omega$ and exhibits strictly subcritical growth. Note that we can find $\varepsilon^{*}>0$ such that $s \mapsto \lambda s-f(x, s)+\varepsilon^{*}|s|^{r-2} s$ is nondecreasing on $\mathbb{R}$ for a.a. $x \in \Omega$.

We have the following multiplicity theorem for problem $(Q)_{\lambda}$.

Theorem 4.2. Let hypotheses $H(f)_{2}$ be satisfied and let $\lambda>\hat{\lambda}_{2}(2), \lambda \notin \hat{\sigma}(2)$, then problem $(Q)_{\lambda}$ has at least four nontrivial solutions whereby two of them have constant sign, namely $u_{0} \in \operatorname{int}\left(C_{0}^{1}(\bar{\Omega})_{+}\right), v_{0} \in-\operatorname{int}\left(C_{0}^{1}(\bar{\Omega})_{+}\right)$, and two of them have changing sign, namely $y_{0}, \hat{y} \in C_{0}^{1}(\bar{\Omega})$.

Proof. Taking into account Theorem 3.8 we already know the existence of three nontrivial solutions

$$
u_{0} \in \operatorname{int}\left(C_{0}^{1}(\bar{\Omega})_{+}\right), v_{0} \in-\operatorname{int}\left(C_{0}^{1}(\bar{\Omega})_{+}\right) \text {, and } y_{0} \in\left[v_{0}, u_{0}\right] \cap \in C_{0}^{1}(\bar{\Omega}) .
$$


Moreover, we may assume that $u_{0}, v_{0}$ are extremal constant sign solutions. In the present case we have $a(\xi)=\|\xi\|^{p-2} \xi+\xi$ for all $\xi \in \mathbb{R}^{N}$. Hence,

$$
\nabla a(\xi)=\|\xi\|^{p-2}\left(I+(p-2) \frac{\xi \otimes \xi}{\|\xi\|^{2}}\right)+I \quad \text { for all } \xi \in \mathbb{R}^{N} \backslash\{0\} .
$$

It follows that

$$
(\nabla a(\xi) y, y)_{\mathbb{R}^{N}} \geq\|y\|^{2} \quad \text { for all } \xi, y \in \mathbb{R}^{N} .
$$

Invoking the tangency principle of Pucci-Serrin [31, Theorem 2.5.2], we have

$$
v_{0}(x)<y_{0}(x)<u_{0}(x) \text { for all } x \in \Omega .
$$

As already observed in Remark 4.1, there exists $\varepsilon^{*}>0$ such that

$$
s \mapsto \lambda s-f(x, s)+\varepsilon^{*}|s|^{r-2} s \text { is nondecreasing on } \mathbb{R} \text { for a.a. } x \in \Omega .
$$

Applying (4.1) and (4.2) we derive

$$
\begin{aligned}
-\Delta_{p} u_{0}(x)-\Delta u_{0}(x)+\varepsilon^{*} u_{0}(x)^{r-1} & =\lambda u_{0}(x)-f\left(x, u_{0}(x)\right)+\varepsilon^{*} u_{0}(x)^{r-1} \\
& \geq \lambda y_{0}(x)-f\left(x, y_{0}(x)\right)+\varepsilon^{*}\left|y_{0}(x)\right|^{r-2} y_{0}(x) \\
& =-\Delta_{p} y_{0}(x)-\Delta y_{0}(x)+\varepsilon^{*}\left|y_{0}(x)\right|^{r-2} y_{0}(x)
\end{aligned}
$$

almost everywhere in $\Omega$. Since $y_{0}, u_{0} \in C_{0}^{1}(\bar{\Omega})$ and (4.1) holds, we infer from Proposition 2.5 that

$$
y_{0} \in \operatorname{int}_{C_{0}^{1}(\bar{\Omega})}\left[v_{0}, u_{0}\right] .
$$

The energy functional of problem $(Q)_{\lambda}$, namely $\varphi_{\lambda}: W_{0}^{1, p}(\Omega) \rightarrow \mathbb{R}$, is defined by

$$
\varphi_{\lambda}(u)=\frac{1}{p}\|\nabla u\|_{L^{p}(\Omega)}^{p}+\frac{1}{2}\|\nabla u\|_{L^{2}(\Omega)}^{2}-\frac{\lambda}{2}\|u\|_{L^{2}(\Omega)}^{2}+\int_{\Omega} F(x, u) d x .
$$

Evidently, $\varphi_{\lambda} \in C^{2}\left(W_{0}^{1, p}(\Omega)\right)$. Let $\beta_{\lambda}: W_{0}^{1, p}(\Omega) \rightarrow \mathbb{R}$ be the functional obtained by truncating the reaction of $(Q)_{\lambda}$ at $\left\{v_{0}(x), u_{0}(x)\right\}$ as in the proof of Proposition 3.7. We have $\beta_{\lambda} \in C^{2-0}\left(W_{0}^{1, p}(\Omega)\right)$ and

$$
\left.\beta_{\lambda}\right|_{\left[v_{0}, u_{0}\right]}=\left.\varphi_{\lambda}\right|_{\left[v_{0}, u_{0}\right]}
$$

(see (3.22)). Both functionals are coercive, consequently they satisfy the PScondition (see Proposition 2.13). We consider the homotopy

$$
h(t, u)=t \beta_{\lambda}(u)+(1-t) \varphi_{\lambda}(u) \quad \text { for all } t \in[0,1] \text { and all } u \in W_{0}^{1, p}(\Omega) .
$$

Suppose that we can find $\left(t_{n}\right)_{n \geq 1} \subseteq[0,1]$ and $\left(u_{n}\right)_{n \geq 1} \subseteq W_{0}^{1, p}(\Omega)$ such that

$$
t_{n} \rightarrow t, u_{n} \rightarrow y_{0} \text { in } W_{0}^{1, p}(\Omega) \quad \text { and } \quad h_{u}^{\prime}\left(t_{n}, u_{n}\right)=0 \quad \text { for all } n \geq 1 .
$$

Then, we have

$$
\Delta_{p} u_{n}+\Delta u_{n}=t_{n} N_{h_{\lambda}}\left(u_{n}\right)+(1-t) N_{f}\left(u_{n}\right)
$$

meaning that

$$
\begin{aligned}
-\Delta_{p} u_{n}-\Delta u_{n} & =t_{n} h_{\lambda}\left(x, u_{n}\right)+(1-t) f\left(x, u_{n}\right) & & \text { in } \Omega, \\
u_{n} & =0 & & \text { on } \partial \Omega,
\end{aligned}
$$

for all $n \geq 1$. From Ladyzhenskaya-Ural'tseva [25, p. 286] we know that the solutions of (4.5) are essentially bounded, i.e., there exists $M_{2}>0$ such that

$$
\left\|u_{n}\right\|_{L^{\infty}(\Omega)} \leq M_{2} \text { for all } n \geq 1 .
$$


Then, the regularity results of Lieberman [26, p. 320] imply the existence of $\gamma \in$ $(0,1)$ and $M_{3}>0$ such that

$$
u_{n} \in C_{0}^{1, \gamma}(\bar{\Omega}) \text { and }\left\|u_{n}\right\|_{C_{0}^{1, \gamma}(\bar{\Omega})} \leq M_{3} \quad \text { for all } n \geq 1 .
$$

Since $C_{0}^{1, \gamma}(\bar{\Omega})$ is compactly embedded in $C_{0}^{1}(\bar{\Omega})$, it follows from (4.4) that

$$
u_{n} \rightarrow y_{0} \text { in } C_{0}^{1}(\bar{\Omega}) \text {. }
$$

Due to (4.3) we conclude that

$$
u_{n} \in\left[v_{0}, u_{0}\right] \backslash\{0\} \quad \text { for all } n \geq n_{0} .
$$

Hence, the sequence $\left(u_{n}\right)_{n \geq n_{0}}$ contains only nodal solutions of $(Q)_{\lambda}$ and from there we are done.

Accordingly, we may assume that there exists $\rho \in(0,1)$ small such that

$$
\bar{B}_{\rho}\left(y_{0}\right) \cap K_{h(t, 0)}=\left\{y_{0}\right\} \quad \text { for all } t \in[0,1]
$$

with

$$
\bar{B}_{\rho}\left(y_{0}\right)=\left\{u \in W_{0}^{1, p}(\Omega):\left\|u-y_{0}\right\| \leq \rho\right\} .
$$

Invoking the homotopy invariance property of critical groups, we have

$$
C_{k}\left(\varphi_{\lambda}, y_{0}\right)=C_{k}\left(\beta_{\lambda}, y_{0}\right) \quad \text { for all } k \geq 0
$$

Owing to the proof of Proposition 3.7 we know that $y_{0}$ is a critical point of $\beta_{\lambda}$ (i.e., $y_{0} \in K_{\beta_{\lambda}}$ ) of mountain pass type. Therefore, due to Chang [10],

$$
C_{1}\left(\beta_{\lambda}, y_{0}\right) \neq 0
$$

which combined with (4.6) yields

$$
C_{1}\left(\varphi_{\lambda}, y_{0}\right) \neq 0
$$

Since $\varphi_{\lambda} \in C^{2}\left(W_{0}^{1, p}(\Omega)\right)$, from (4.7) and Papageorgiou-Smyrlis [30], we have

$$
C_{k}\left(\varphi_{\lambda}, y_{0}\right)=\delta_{k, 1} \mathbb{Z} \quad \text { for all } k \geq 0
$$

which thanks to (4.6) gives

$$
C_{k}\left(\beta_{\lambda}, y_{0}\right)=\delta_{k, 1} \mathbb{Z} \quad \text { for all } k \geq 0 .
$$

Because of hypothesis $\lambda>\hat{\lambda}_{2}(2), \lambda \notin \hat{\sigma}(2)$, there is a number $m \geq 2$ such that $\lambda \in\left(\hat{\lambda}_{m}(2), \hat{\lambda}_{m+1}(2)\right)$. Let $\varepsilon\left(0, \hat{\lambda}_{m}(2)\right)$. By virtue of hypothesis $\mathrm{H}(\mathrm{f})_{2}(\mathrm{iii})$, we find $\delta=\delta(\varepsilon)>0$ such that

$$
|f(x, s)| \leq \varepsilon|s| \text { for a.a. } x \in \Omega \text { and for all }|s| \leq \delta .
$$

It follows that

$$
F(x, s) \leq \frac{\varepsilon}{2} s^{2} \quad \text { for a.a. } x \in \Omega \text { and for all }|s| \leq \delta .
$$

Let $\Theta_{\lambda}: W_{0}^{1, p}(\Omega) \rightarrow \mathbb{R}$ be the $C^{2}$-functional defined by

$$
\Theta_{\lambda}(u)=\frac{1}{p}\|\nabla u\|_{L^{p}(\Omega)}^{p}+\frac{1}{2}\|\nabla u\|_{L^{2}(\Omega)}^{2}-\frac{\lambda}{2}\|u\|_{L^{2}(\Omega)}^{2} .
$$

Apparently, $\Theta$ is coercive, hence, it satisfies the PS-condition. Then, from (4.9) and Chang [10, Corollary 5.1.25 (p. 336)], we verify that

$$
C_{k}\left(\varphi_{\lambda}, 0\right)=C_{k}\left(\Theta_{\lambda}, 0\right) \quad \text { for all } k \geq 0,
$$


which implies (see (4.6)) that

$$
C_{k}\left(\beta_{\lambda}, 0\right)=C_{k}\left(\Theta_{\lambda}, 0\right) \quad \text { for all } k \geq 0 .
$$

By reason of $\lambda \in\left(\hat{\lambda}_{m}(2), \hat{\lambda}_{m+1}(2)\right)$ and due to Cingolani-Vannella [13, Theorem 1.1] one has

$$
C_{k}\left(\Theta_{\lambda}, 0\right)=\delta_{k, d_{m}} \mathbb{Z} \quad \text { for all } k \geq 0,
$$

where $d_{m}=\operatorname{dim} \bigoplus_{i=1}^{m} E\left(\hat{\lambda}_{i}(2)\right) \geq 2$. Owing to (4.10) we derive that

$$
C_{k}\left(\beta_{\lambda}, 0\right)=\delta_{k, d_{m}} \mathbb{Z} \quad \text { for all } k \geq 0 .
$$

From the proof of Proposition 3.7 (see Claim 2), we know that $u_{0}, v_{0}$ are both local minimizers of $\beta_{\lambda}$. Hence

$$
C_{k}\left(\beta_{\lambda}, u_{0}\right)=C_{k}\left(\beta_{\lambda}, v_{0}\right)=\delta_{k, 0} \mathbb{Z} \text { for all } k \geq 0 .
$$

Recalling that $\beta_{\lambda}$ is coercive, it follows that

$$
C_{k}\left(\beta_{\lambda}, \infty\right)=\delta_{k, 0} \mathbb{Z} \quad \text { for all } k \geq 0 .
$$

Suppose that $K_{\beta_{\lambda}}=\left\{0, u_{0}, v_{0}, y_{0}\right\}$. Then, from (4.8), (4.11), (4.12), (4.13) and the Morse relation (2.6) with $t=-1$, we have

$$
2(-1)^{0}+(-1)^{1}+(-1)^{d_{m}}=(-1)^{0},
$$

which gives $(-1)^{d_{m}}=0$, a contradiction. Therefore, we can find $\hat{y} \in K_{\beta_{\lambda}}, \hat{y} \notin$ $\left\{0, u_{0}, v_{0}, y_{0}\right\}$. Since $K_{\beta_{\lambda}} \subseteq\left[v_{0}, u_{0}\right]$ (see the proof of Claim 1 in the proof of Proposition 3.7), one gets $\hat{y} \in\left[v_{0}, u_{0}\right] \backslash\{0\}$. The extremal property of $u_{0}$ and $v_{0}$ implies that $\hat{y}$ has changing sign. The nonlinear regularity theory (see [25], [26]) yields $\hat{y} \in C_{0}^{1}(\bar{\Omega})$. That finishes the proof.

\section{ACKNOWLEDGEMENT}

The authors wish to thank a knowledgeable referee for his/her suggestions and remarks.

\section{REFERENCES}

[1] S. Aizicovici, N. S. Papageorgiou, and V. Staicu, Degree theory for operators of monotone type and nonlinear elliptic equations with inequality constraints, Mem. Amer. Math. Soc. 196 (2008), no. 915.

[2] A. Ambrosetti and D. Lupo, On a class of nonlinear Dirichlet problems with multiple solutions, Nonlinear Anal. 8 (1984), no. 10, 1145-1150.

[3] A. Ambrosetti and G. Mancini, Sharp nonuniqueness results for some nonlinear problems, Nonlinear Anal. 3 (1979), no. 5, 635-645.

[4] D. Arcoya and D. Ruiz, The Ambrosetti-Prodi problem for the p-Laplacian operator, Comm. Partial Differential Equations 31 (2006), no. 4-6, 849-865.

[5] D. Averna, S. A. Marano, and D. Motreanu, Multiple solutions for a Dirichlet problem with p-Laplacian and set-valued nonlinearity, Bull. Aust. Math. Soc. 77 (2008), no. 2, 285-303.

[6] V. Benci, P. D'Avenia, D. Fortunato, and L. Pisani, Solitons in several space dimensions: Derrick's problem and infinitely many solutions, Arch. Ration. Mech. Anal. 154 (2000), no. 4, 297-324.

[7] H. Brezis and L. Nirenberg, $H^{1}$ versus $C^{1}$ local minimizers, C. R. Acad. Sci. Paris Sér. I Math. 317 (1993), no. 5, 465-472.

[8] S. Carl and D. Motreanu, Constant-sign and sign-changing solutions for nonlinear eigenvalue problems, Nonlinear Anal. 68 (2008), no. 9, 2668-2676. 
[9] L. Čaklović, S. J. Li, and M. Willem, A note on Palais-Smale condition and coercivity, Differential Integral Equations 3 (1990), no. 4, 799-800.

[10] K.-C. Chang, "Methods in Nonlinear Analysis", Springer-Verlag, Berlin, 2005.

[11] L. Cherfils and Y. Il'yasov, On the stationary solutions of generalized reaction diffusion equations with $p \& q$-Laplacian, Commun. Pure Appl. Anal. 4 (2005), no. 1, 9-22.

[12] S. Cingolani and M. Degiovanni, Nontrivial solutions for $p$-Laplace equations with right-hand side having p-linear growth at infinity, Comm. Partial Differential Equations 30 (2005), no. 7-9, 1191-1203.

[13] S. Cingolani and G. Vannella, Critical groups computations on a class of Sobolev Banach spaces via Morse index, Ann. Inst. H. Poincaré Anal. Non Linéaire 20 (2003), no. 2, 271-292.

[14] P. Clément, M. García-Huidobro, R. Manásevich, and K. Schmitt, Mountain pass type solutions for quasilinear elliptic equations, Calc. Var. Partial Differential Equations 11 (2000), no. 1, 33-62.

[15] M. Cuesta, D. de Figueiredo, and J.-P. Gossez, The beginning of the Fučik spectrum for the $p$-Laplacian, J. Differential Equations 159 (1999), no. 1, 212-238.

[16] E. N. Dancer, Counterexamples to some conjectures on the number of solutions of nonlinear equations, Math. Ann. 272 (1985), no. 3, 421-440.

[17] J. I. Díaz and J. E. Saá, Existence et unicité de solutions positives pour certaines équations elliptiques quasilinéaires, C. R. Acad. Sci. Paris Sér. I Math. 305 (1987), no. 12, 521-524.

[18] N. Dunford and J. T. Schwartz, "Linear Operators. I. General Theory", Interscience Publishers, Inc., New York, 1958.

[19] M. Filippakis, A. Kristály, and N. S. Papageorgiou, Existence of five nonzero solutions with exact sign for a p-Laplacian equation, Discrete Contin. Dyn. Syst. 24 (2009), no. 2, 405-440.

[20] M. E. Filippakis, D. O'Regan and N. S. Papageorgiou, Positive solutions and bifurcation phenomena for nonlinear elliptic equations of logistic type: the superdiffusive case, Commun. Pure Appl. Anal. 9 (2010), no. 6, 1507-1527.

[21] J. P. García Azorero, I. Peral Alonso, and J. J. Manfredi, Sobolev versus Hölder local minimizers and global multiplicity for some quasilinear elliptic equations, Commun. Contemp. Math. 2 (2000), no. 3, 385-404.

[22] L. Gasiński and N. S. Papageorgiou, "Nonsmooth Critical Point Theory and Nonlinear Boundary Value Problems", Chapman \& Hall/CRC, Boca Raton, FL, 2005.

[23] L. Gasiński and N. S. Papageorgiou, "Nonlinear Analysis", Chapman \& Hall/CRC, Boca Raton, FL, 2006.

[24] M. E. Gurtin and R. C. MacCamy, On the diffusion of biological populations, Math. Biosci. 33 (1977), no. 1-2, 35-49.

[25] O. A. Ladyzhenskaya and N. N. Ural'tseva, "Linear and Quasilinear Elliptic Equations", Academic Press, New York, 1968.

[26] G. M. Lieberman, The natural generalization of the natural conditions of Ladyzhenskaya and Ural'tseva for elliptic equations, Comm. Partial Differential Equations 16 (1991), no. 2-3, 311-361.

[27] E. Medeiros and K. Perera, Multiplicity of solutions for a quasilinear elliptic problem via the cohomological index, Nonlinear Anal. 71 (2009), no. 9, 3654-3660.

[28] D. Motreanu and N. S. Papageorgiou, Multiple solutions for nonlinear Neumann problems driven by a nonhomogeneous differential operator, Proc. Amer. Math. Soc. 139 (2011), no. 10, 3527-3535.

[29] E. H. Papageorgiou and N. S. Papageorgiou, A multiplicity theorem for problems with the p-Laplacian, J. Funct. Anal. 244 (2007), no. 1, 63-77.

[30] N. S. Papageorgiou and G. Smyrlis, On nonlinear nonhomogeneous resonant Dirichlet equations, Pacific J. Math. 264 (2013), no. 2, 421-453.

[31] P. Pucci and J. Serrin, "The Maximum Principle", Birkhäuser Verlag, Basel, 2007.

[32] M. Struwe, A note on a result of Ambrosetti and Mancini, Ann. Mat. Pura Appl. (4) 131 (1982), 107-115.

[33] M. Struwe, "Variational Methods", Springer-Verlag, Berlin, 2008.

[34] M. Sun, Multiplicity of solutions for a class of the quasilinear elliptic equations at resonance, J. Math. Anal. Appl. 386 (2012), no. 2, 661-668.

[35] P. Winkert, Local $C^{1}(\bar{\Omega})$-minimizers versus local $W^{1, p}(\Omega)$-minimizers of nonsmooth functionals, Nonlinear Anal. 72 (2010), no. 11, 4298-4303. 
(N. S. Papageorgiou) National Technical University, Department of Mathematics, Zografou Campus, Athens 15780, Greece

E-mail address: npapg@math.ntua.gr

(P. Winkert) Technische Universität Berlin, Institut für Mathematik, Strasse des 17. Juni 136, 10623 Berlin, Germany

E-mail address: winkert@math.tu-berlin.de 